\title{
Suppressed radio emission in supercluster galaxies: enhanced ram pressure in merging clusters?
}

\author{
Jean-Christophe Mauduit ${ }^{1}$, Gary A. Mamon ${ }^{1,2}$ \\ 1 Institut d'Astrophysique de Paris (UMR 7095: CNRS and Univ. Pierre \& Marie Curie), 98 bis Bd. Arago, F-75014 Paris, France \\ 2 GEPI (UMR 8111: CNRS and Univ. Denis Diderot), Observatoire de Paris, F-92195 Meudon, Cédex, France
}

Received 26 April 2007 / Accepted 31 August 2007

\begin{abstract}
Context. Continuum radio emission of galaxies is related to AGN activity and to starbursts, both of which require a supply of gas, respectively to the central black hole and to molecular clouds.

Aims. The environmental influence on the $21 \mathrm{~cm}(1.4 \mathrm{GHz})$ continuum radio emission of galaxies is analyzed in a $600 \mathrm{deg}^{2}$ region of the local Universe containing the Shapley Supercluster (SSC), whose core is thought to be the site of cluster-cluster merging.

Methods. Galaxies in the FLASH and 6dFGS optical/NIR redshift surveys are cross-identified with NVSS radio sources, selected in a subsample doubly complete in volume and luminosity, and classified as starbursts or AGN according to their radio luminosity. We study radio luminosities as well as radio loudness (luminosities normalized by stellar mass) $\mathcal{R}_{\mathcal{K}}$. Environmental effects are studied through a smoothed density field (normalized to that obtained from random catalogs with the same survey edges and redshift selection function) and through the projected distance to the nearest cluster (in units of its virial radius, whose relation to the aperture velocity dispersion is quantified).

Results. The fraction of high $\mathcal{R}_{K}$ galaxies in the dense $10 \mathrm{Mpc}$ Abell 3558 cluster complex at the core of the SSC (SSC-CR) is half as large than elsewhere. Moreover, radio loudness in the SSC-CR is anti-correlated with the density of the large-scale environment and correlated with clustercentric radius: central brightest cluster galaxies (BCGs) in the SSC-CR are an order of magnitude less radioloud than BCGs elsewhere, with signs of suppressed radio loudness in the SSC-CR also present beyond the BCGs, out to at least $0.3 r_{200}$. This correlation is nearly as strong as the tight correlation of $K$-band luminosity, $L_{K}$, with clustercentric radius ( $K$-luminosity segregation), inside the SSC-CR, with a mild $K$-luminosity segregation outside the SSC-CR.

Conclusions. The suppression of radio loudness in SSC-CR BCGs can be attributed to cluster-cluster mergers that destroy the cool core and thus the supply of gas to the central AGN. We analytically demonstrate that the low radio loudness of non-BCG galaxies within SSC-CR clusters cannot be explained by direct major galaxy mergers or rapid galaxy flyby collisions, but by the loss of gas supply through the enhanced ram pressure felt when these galaxies cross the shock front between the two merging clusters and are afterwards subjected to the stronger wind from the second cluster.
\end{abstract}

Key words. galaxies: active - galaxies: starburst - galaxies: clusters: general - (cosmology:) large-scale structure of Universe

\section{Introduction}

Galaxies are not isolated objects and evolve in tight relation to the structure they belong to. These environmental effects are expected to hold even more strongly for galaxies with important emission in the radio continuum, i.e. radio galaxies. Such galaxies range from the less radio luminous ones, star-forming galaxies (hereafter SFGs), to the highest radio-luminous ones, galaxies with an active galactic nucleus (AGN). However, the exact role of the environment on galaxy activity - starburst or nuclear - is still not clear.

The first environmental studies of radio galaxies were based upon small samples of galaxies distributed over the entire sky to derive their clustering properties. In a pioneering study, Dressler et al. (1985) showed that low luminosity radio galaxies (i.e. SFGs) avoid dense environments. Peacock \& Nicholson (1991) found that radio galaxies are more strongly clustered than individual galaxies but less clustered than clusters of galaxies. Galaxies of intermediate radio luminosity tend to lie in poor groups (Bahcall \& Chokshi 1992). The most powerful radio sources appear to favor galaxy groups and poor clusters (Prestage \& Peacock 1988; Hill \& Lilly 1991).

Send offprint requests to: Gary Mamon, email: gam AT iap.fr
In recent years, the advent of large radio and optical catalogs of galaxies has permitted a better statistical characterization of these trends. Large-scale studies have matched the angular positions of radio galaxies in the NRAO VLA Sky Survey (NVSS, Condon et al. 1998) and Faint Images of the Radio Sky at Twenty Centimeters (FIRST, Becker et al. 1995) with the redshift-space positions of optical galaxies in the $2 \mathrm{dF}$ Galaxy Redshift Survey (2dFGRS, Colless et al. 2001) or the Sloan Digitized Sky Survey (SDSS, Strauss et al. 2002). Many of the previous trends noted above have been confirmed and developed. Magliocchetti et al. (2004) showed that AGN appear to be strongly clustered and find no significant differences in the clustering of faint and luminous radio galaxies. Best (2004) found that the fraction of SFGs (defined as galaxies of low radio luminosity) decreases with the density of the environment and that AGN are preferentially located in groups and poor to moderate richness clusters and avoid regions of very low density. In compact groups of galaxies, the SFG fraction increases drastically from the core to the halo while AGN seem to always lie in the cores (Coziol et al. 1998). In clusters too, SFGs are broadly distributed, whereas AGN are centrally concentrated (Miller \& Owen 2002). However, AGN that are brightest cluster galaxies (BCGs) have the same distribution of radio luminosi- 
ties as non-BCGs (Best et al. 2007). All these studies seem to indicate that radio galaxies, in general, prefer environments of intermediate density, with different behaviors at both ends of the radio luminosity distribution, the lowest radio emission avoiding dense environments and the most powerful ones avoiding very low density regions.

Since the fraction of radio galaxies (both SFGs and AGN) seem to depend more on the large-scale environment than on the small-scale one (Best 2004), it is worth investigating the properties of radio galaxies on the scales of superclusters. Indeed, these superstructures contain a wide variety of environments, from small galaxy groups to big clusters. It is be therefore interesting to mix the two approaches (the large-scale and local environment) over an area containing diverse environments, from empty regions (voids) to the densest ones (a supercluster).

In the present work, we study the effect of the supercluster environment on the radio luminosity and $K$-band normalized radio loudness of galaxies. For this, we analyze the deepest, homogeneous redshift sample of radio-emitting galaxies covering the Shapley Supercluster and its surroundings in a region $70^{\circ} \times 10^{\circ}$.

The Shapley Supercluster (SSC) is the densest region in the local universe (Raychaudhury et al. 1991; Fabian 1991). The SSC and some of its clusters have been studied in detail over the years, mainly by Quintana et al. (1995, 2000), Bardelli et al. (2000, 2001) and Drinkwater et al. (2004) and its radio properties were first investigated by Venturi et al. (1997). Moreover, this region is interesting because it hosts a variety of environments. In particular, its central, $10 \mathrm{Mpc}$ radius region, known as Shapley 8 or the Abell 3558 (A3558) cluster complex (hereafter SSC-CR following the nomenclature of Quintana et al. 2000), is thought to be globally undergoing gravitational collapse (Reisenegger et al. 2000), with its central clusters, Abell 3556, 3558 and 3562 possibly merging today with one another and with smaller galaxy groups (Bardelli et al. 2000; Venturi et al. 2000).

Several authors recently pointed out that cluster-cluster merging can have dramatic consequences on radio galaxies and might be the key to the general trends observed. But these consequences remain uncertain. For example, Owen et al. (1999) and Miller \& Owen (2003) found an increase of star-formation and AGN activity that might be enhanced in several Abell clusters by an ongoing merger with another cluster. The dense regions of superclusters are expected to be an ideal laboratory for studying colliding clusters. In the SSC-CR, Venturi et al. (2000) found that the A3558 cluster presents a deficit of radio galaxies. On the other hand, another cluster complex outside the SSC-CR, centered around Abell 3528 (A3528), appears to have different physical characteristics, as Venturi et al. (2001) found the A3528 complex to be active at radio wavelengths, although its radio luminosity function turns up being similar with the generic one derived by Ledlow \& Owen (1996). Bardelli et al. (2001) argued that this complex was possibly in a pre-merger state.

Cross-identification with NVSS radio sources gives us access, not only to the radio luminosity but also to the radioloudness (here defined as power normalized to stellar mass from $K$-band luminosity), a variable that has been generally overlooked in previous studies.

The merging of the optical, near-infrared (NIR) and radio catalogs is described in Sect. 2. In Sect. 3, we compare the distributions of radio luminosity and of radio loudness between the A3558 complex, the A3528 complex and the remaining FLASH region. We study how the radio power and loudness are modulated by the density of the large-scale environment in Sect. 4 and with the clustercentric radius in Sect. 5. We compare our results with other studies in Sect. 6, and discuss them in Sect. 7, making qualitative and semi-quantitative predictions of the effects of galaxy mergers, rapid flyby collisions and altered ram pressure stripping on galaxies within colliding and merging clusters. In two appendices, we compute the relation between aperture velocity dispersion and virial radius for a Navarro, Frenk, \& White (1996, hereafter NFW) model and the rate of direct galaxy mergers in overlapping colliding clusters of equal mass. A summary is provided in Sect. 8 . Throughout this paper the values adopted for the cosmological parameters are $\Omega_{m}=0.3, \Omega_{\Lambda}=0.7$ and a Hubble constant $H_{0}=70 \mathrm{~km} \mathrm{~s}^{-1} \mathrm{Mpc}^{-1}$.

\section{Catalogs}

\subsection{Redshift catalogs}

We cross-correlate radio-detected galaxies from the NVSS radio survey with galaxies from two different optical/NIR surveys: the FLAir Shapley-Hydra survey (FLASH) and the Six degree Field Galaxy Survey (6dFGS).

The FLASH survey (Kaldare et al. 2003), is a redshift survey containing 3141 galaxies with measured redshifts in a $70^{\circ} \times$ $10^{\circ}$ strip, aligned in galactic coordinates $\left(260^{\circ}<\ell<330^{\circ}\right.$ and $25^{\circ}<b<35^{\circ}$ ), extending from the Shapley Supercluster (SSC) to the Hydra cluster, covering a solid angle of $605 \mathrm{deg}^{2}=$ 0.184 sr. Its input catalog is the Hydra-Centaurus Catalogue of Raychaudhury (1990), a photometric catalog down to $b_{J}=$ 17, compiled by scanning UKST Southern Sky Survey plates with the Automated Photographic Measuring (APM) facility in Cambridge. Absolute positions are accurate to $\sim 1^{\prime \prime}$. The FLASH survey magnitude limit, after correction for Galactic extinction, is $b_{J}=16.7$. Radial velocities were obtained with the FLAIR-II spectrograph mounted at the UKST. Additional data from the literature (NED, ZCAT) was incorporated into the original FLAIR redshift sample. The rms error in the redshifts is $95 \mathrm{~km} \mathrm{~s}^{-1}$. Kaldare et al. (2003) assert that the only redshift bias in their sample is due to magnitude-dependent incompleteness, which is $50 \%$ at the $b_{J}=16.7$ survey limit. The overall spectroscopic completeness of the catalog is of $68 \%$ with redshifts for 3141 of the 4613 galaxies in the catalog. The catalog median depth is $\sim 10000 \mathrm{~km} \mathrm{~s}^{-1}$.

We combine this survey with the 6dFGS survey (Jones et al. 2004) Data Release 2. 2 As its principal input catalog, 6dFGS uses the $K$-band galaxies from the Two Micron All Sky Survey (2MASS, Jarrett et al. 2000) and includes all galaxies brighter than $K_{\text {tot }}=12.75$ (corresponding to roughly the same $b_{J}$ limit as that of the FLASH survey). Contrary to optically selected galaxies, $K$-band selected galaxy samples are not biased towards recent star formation activity, and the low extinction in the $K$ band ensures a deeper view through the interstellar dust of the observed galaxies (as well as through our Milky Way). 6dFGS velocities were obtained with the robotized $6 \mathrm{dF}$ spectrograph mounted at the UKST (in replacement of FLAIR II). We retrieve the 6dFGS galaxies with a redshift quality factor of 4 (reliable redshift) in the FLASH area. The median depth of the catalog is $\langle c z\rangle=16008 \mathrm{~km} \mathrm{~s}^{-1}$ and the error on $Q=4$ velocities is $46 \mathrm{~km} \mathrm{~s}^{-1}$. In this catalog, a galaxy may appear several times due to multiple observations. In order to have a unique sample of 6dFGS galaxies, we replace multiple redshift measurements by their mean.

\footnotetext{
1 The FLASH survey catalog was obtained via the VizieR On-line Data Catalog: J/MNRAS/339/652.

2 The 6dFGS Second Data Release is publicly available from the 6dFGS website at http://www.mso.anu.edu.au/6dFGS/
} 
We merge these two redshift catalogs by cross-identifying them with a search radius of $6^{\prime \prime}$, keeping the (more precise) 6dFGS velocity for matching pairs and adding non-matched FLASH galaxies to our catalog. As this work reached completion, a new set of velocities has been published by Proust et al. (2006), which goes to fainter magnitudes in some regions. However, we chose not to use these velocities because of the inhomogeneous selection and smaller solid angle of this data set. In the end, our "6dFGS+FLASH catalog" consists of 5132 galaxies with redshifts in the FLASH region.

\subsection{Radio catalog}

The NVSS is a radio continuum survey at frequency $1.4 \mathrm{GHz}$ $(21 \mathrm{~cm})$ covering the sky north of $-40^{\circ}$, thus fully overlapping the FLASH area 3 The source catalog (Condon et al. 1998) contains $1.8 \times 10^{6}$ entries. It has an angular resolution of $45^{\prime \prime}$ (FWHM). Condon et al. have estimated the differential completeness to be $99 \%$ at flux $S_{1.4 \mathrm{GHz}}=3.5 \mathrm{mJy}$, and from their analysis we infer a differential completeness of $75 \%$ at $2.8 \mathrm{mJy}$. Selecting the sources in the FLASH area, we retrieve $\sim 68000$ radio sources. Although the FIRST survey is a similar survey with a better angular resolution of 5" and a completeness of 95\% at $S_{1.4 \mathrm{GHz}}>2 \mathrm{mJy}$, it does not cover further South than $-10^{\circ}$ in declination, which is not enough to reach the SSC region.

\subsection{Merged radio-NIR galaxy sample and radio galaxy classification}

We cross-identify the the 6dFGS+FLASH galaxies with the NVSS radio sources using a conservative search radius of $15^{\prime \prime}$, $1 / 3$ of the NVSS FWHM resolution. We find 810 matches with recession velocities $v<30000 \mathrm{~km} \mathrm{~s}^{-1}$. To avoid selection effects, we then work with a subsample that is flux-limited to

$S_{1.4 \mathrm{GHz}} \geq S_{\min }=2.8 \mathrm{mJy}$,

(for 75\% differential completeness) and volume-limited to a section of a wide shell, where the SSC lies at the mean depth:

$10000<v<v_{\max }=18800 \mathrm{~km} \mathrm{~s}^{-1}$.

For a complete selection in radio luminosity with a maximal number of radio-detected galaxies selected, we adopt a lower limit to the radio-luminosity of

$h_{70}^{2} \log L_{1.4 \mathrm{GHz}}>h_{70}^{2} \log L_{1.4 \mathrm{GHz}}\left(S_{\min }, v_{\max } / c\right)=22.41$,

with $L_{1.4 \mathrm{GHz}}(S, z)=4 \pi D_{L}^{2}(z) S(1+z)^{-(\alpha+1)}$, where $D_{L}(z)$ is the cosmological luminosity distance at redshift $z=v / c, c$ is the velocity of light, and the term $(1+z)^{-(\alpha+1)}$ is the product of the $k$-correction for a power-law radio spectrum: $S(v) \propto v^{\alpha}$, with $\alpha \simeq-0.7$ (e.g Condon 1992) and the redshift correction for the frequency normalization 44 In the velocity range of equation (2) lie 2363 galaxies in the merged 6dFGS+FLASH catalog, which

\footnotetext{
${ }^{3}$ A text version of the NVSS catalog, NVSSCatalog.text, is available by ftp at nvss.cv.nrao.edu. It was generated with NVSSlist version 2.17 (August 2001 B. Cotton) on the entire FITS database.

4 Peculiar velocities make the radial velocity an imperfect distance estimator. For clusters with velocity dispersions as high as $800 \mathrm{~km} \mathrm{~s}^{-1}$, typical distance errors will be $800 / 14500=5 \%$ and can be as high as $14 \%$ for peculiar velocities as large as 2.5 cluster velocity dispersions. Therefore, the errors in relative luminosity will typically be $5 \%$, and can easily be neglected given the several orders of magnitude range in radio luminosities (See Fig. 1).
}

we will refer to as our "galaxy sample". Among those galaxies, 142 galaxies ( $\sim 6 \%$ of our galaxy sample) show radio emission above the lower radio flux limit of equation (1) and radio luminosity limit of equation (3). $85 \%$ of the sources lie within a radius of $10^{\prime \prime}$.

Following Sadler et al. (2002), we define high radio luminosity galaxies as active galactic nuclei (AGN), with lower radio luminosity galaxies as star forming galaxies (SFGs), and adopt their minimum AGN radio luminosity of

$\log L_{1.4 \mathrm{GHz}}=23.05$,

(after correction to our adopted Hubble constant). Note that Machalski \& Godlowski (2000) find that galaxies with AGN spectra predominate starting at $\log L_{1.4 \mathrm{GHz}}=23.31$ (LCRS survey) and 23.05 (UGC galaxies, again converting both to our adopted Hubble constant). Both Machalski \& Godlowski and Sadler et al. find AGN with radio luminosities as low as $10^{22} \mathrm{~W} \mathrm{~Hz}^{-1}$ Interestingly, our lower luminosity cutoff at $\log L_{1.4 \mathrm{GHz}}=22.41$ corresponds to roughly $15 \mathrm{M}_{\odot} \mathrm{yr}^{-1}$ - using $\operatorname{SFR}\left(\mathrm{M}_{\odot} \mathrm{yr}^{-1}\right)=5.9 \pm 1.8 \times 10^{-22} L_{1.4 \mathrm{GHz}}\left(\mathrm{W} \mathrm{Hz}^{-1}\right)$, following Yun et al. (2001) - which is close to the approximate lower limit for the definition of a starburst galaxy (SBG). According to this classification, there are 114 SBGs and 28 AGN (respectively $\sim 5 \%$ and $1 \%$ of our galaxy sample) among the 142 radiodetected galaxies. Figure 1 shows our selection as a function of radio luminosity and radial velocity (roughly equivalent to distance), and highlights our subsample of radio-detected galaxies with the constraints on radial velocity (eq. [2]) and radio luminosity (eq. [3]), i.e. doubly complete in volume and radio luminosity.

In addition, we visually checked all identifications using radio contours from NVSS, overlaid on optical images from the Digitized Sky Survey (DSS). In only a very few cases were the identifications not clear. However, we are probably missing a few AGN because the radio emission from their lobes could be too far from the optical position of the galaxy to result in a match. Because our visual checks indicate that gross misidentifications are rare, we expect that these more subtle misidentifications should also be infrequent in our final sample, which should therefore be valid to perform a statistical analysis.

Throughout the paper, we will consider both radio luminosity and radio loudness 5 . We use the $K$-band luminosity as a tracer of the galaxy mass, and therefore define the dimensionless radio loudness as

$$
\begin{aligned}
\log \left(\mathcal{R}_{K}\right) & =\log \left(\frac{S_{1.4 \mathrm{GHz}}}{1 \mathrm{mJy}}\right)+0.4 K-5.82 \\
& =\log \left(\frac{L_{1.4 \mathrm{GHz}}}{1 \mathrm{~W} \mathrm{~Hz}^{-1}}\right)+0.4 M_{K}-12.90,
\end{aligned}
$$

where we used the normalization of $666.7 \mathrm{Jy}$ for an object with $K=0$ (Cutri et al. 2006). $K$-band magnitudes were retrieved from the 2MASS Extended Source catalog for all the galaxies in our sample and $K$-band luminosities derived using the FLASH and 6dFGS redshifts. We computed $k$ corrections with the approximation $k_{K}=-1.688 z+3.458 z^{2}$, which appears to be a good fit to better than 0.01 mag for $z<0.1$ to the tabulated Sa galaxy $k$-corrections of Poggianti (1997). The FLASH and 6dFGS surveys also provide $b_{J}$ magnitudes, measured with the APM and SuperCosmos machines, respectively. We $k$-corrected them with $k_{b_{J}}=4.04 z+2.00 z^{2}$, again a fit (better than 0.001

\footnotetext{
5 Since we restrict our analysis only to the subsample of radio emitting galaxies, the adjective radio-loud will refer to high $\mathcal{R}_{K}$.
} 


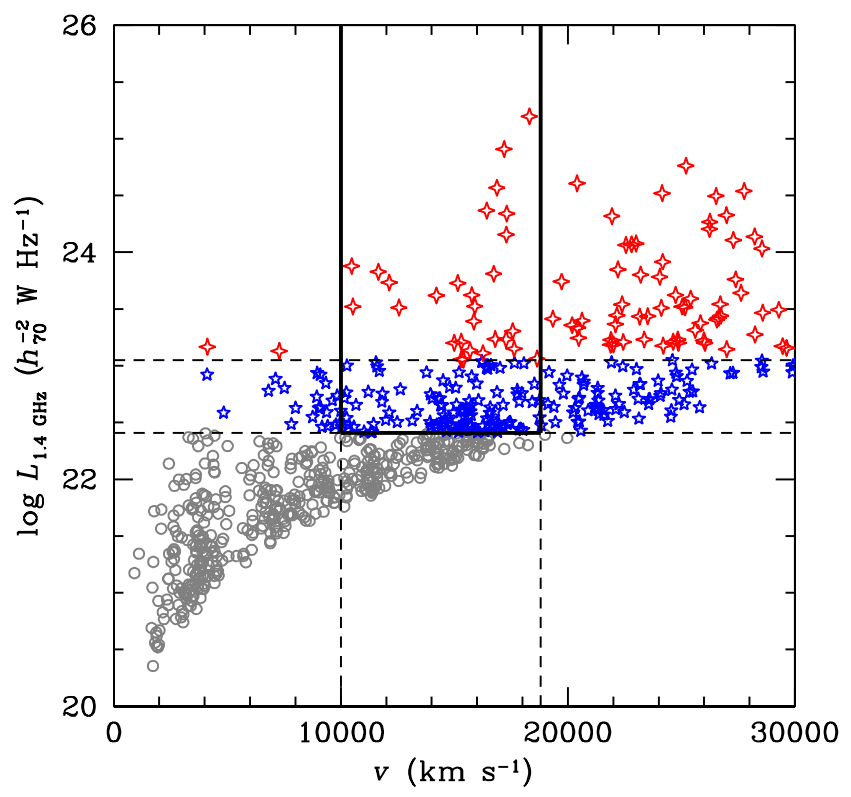

Fig. 1. Radio luminosity versus radial velocity (all radiodetected galaxies with $v<30000 \mathrm{~km} \mathrm{~s}^{-1}$ are shown). AGN are the small red open crosses, SBGs are the blue stars and SFGs the gray circles. Horizontal lines delineate the limits for SBGs (eq. [3]) and AGN, while the vertical lines delineate our region of analysis (eq. [2]). The $U$-shaped set of solid line segments delimits our subsample of radio-detected galaxies that is doubly complete in volume and radio luminosity.

mag for $z<0.1)$ to Poggianti's $k$-corrections for an Sa galaxy in the nearby $B$ band.

Because $K$-band luminosity is closely (but not perfectly) related to stellar mass, $\mathcal{R}_{K}$ can be thought a measure of stellar mass-weighted efficiency of instantaneous star formation for SBGs. For AGN, which usually have higher radio to optical ratios, it gives an idea of the radio power of the nuclei in comparison to the stellar mass of the galaxy.

\subsection{Large scale structure}

The wedge plot of Figure 2 shows the three dimensional distribution of galaxies in our 6dFGS+FLASH catalog, projected in galactic longitude. This region, which spans $36 \mathrm{Mpc}$ by $251 \mathrm{Mpc}$ in galactic coordinates, is filled with 19 Abell clusters of galaxies in the velocity range of equation (2). The SSC is the large overdensity centered on A3558 $\left(13^{h} 27^{m},-31^{\circ} 29^{\prime}, v=\right.$ $14390 \mathrm{~km} \mathrm{~s}^{-1}$ ), with the filaments extending away.

In this paper, we define the SSC-CR (or the A3558 cluster complex) as a $10 \mathrm{Mpc}$ radius cone, limited to velocities within $3 \sigma_{v}$ of the set of clusters A3556, A3558, A3559, A3560 and A3562 that compose it $\left(11459 \mathrm{~km} \mathrm{~s}^{-1}<\mathrm{v}<17545 \mathrm{~km} \mathrm{~s}^{-1}\right)$. The complex has a dense core with clusters A3562, A3558, A3556 and groups SC 1329-312, SC 1327-313, with clusters A3559 and A3560 in its envelope. Altogether, we have 423 galaxies in the SSC-CR, among which 20 SBGs, but not a single AGN. Another prominent feature to the West of the supercluster is the A3528 cluster complex, centered on A3528 at $\left(12^{h} 54^{m},-29^{\circ} 01^{\prime}, v=\right.$ $15829 \mathrm{~km} \mathrm{~s}^{-1}$ ). The A3528 complex is defined in a similar manner as the SSC-CR: a cone of radius $10 \mathrm{Mpc}$, with velocities within $3 \sigma_{v}^{\mathrm{A} 3528}: 13069 \mathrm{~km} \mathrm{~s}^{-1}<\mathrm{v}<18771 \mathrm{~km} \mathrm{~s}^{-1}$. It thus

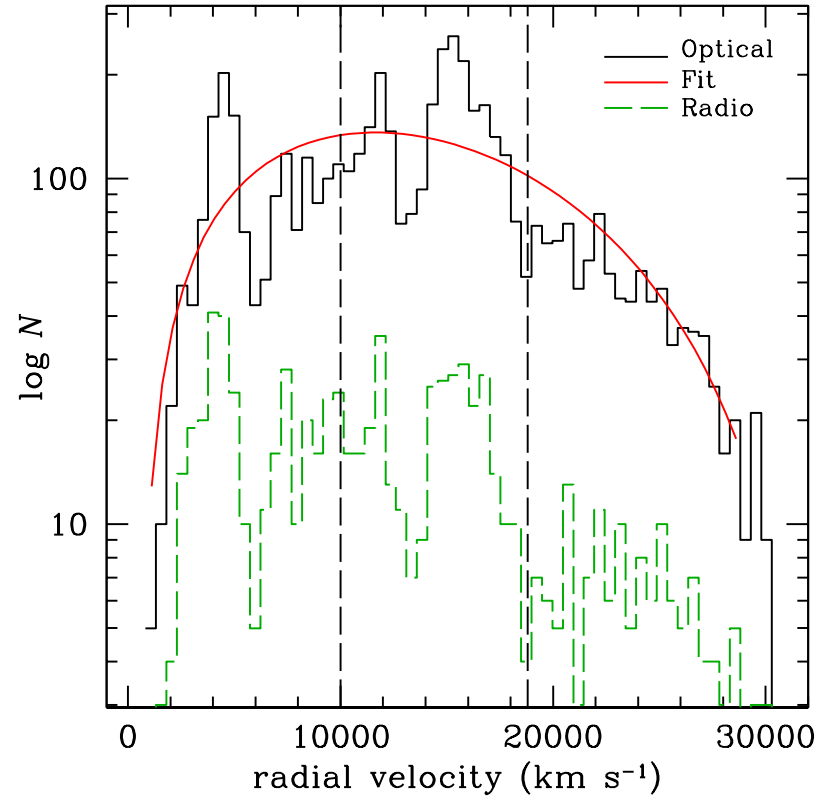

Fig. 3. Distribution of radial velocities of the optical/NIR (solid black histogram) and radio (green dashed histogram) samples. The two dashed vertical lines delineate our velocity cuts (corresponding to the rough velocity limits of the SSC). The red curve is the 4th order polynomial fitted to the underlying optical/NIR distribution and represent the empirical selection function of the data.

contains clusters A3528, A3530 and A3532. We have 121 confirmed galaxies in this complex, among which 5 SBGs and 5 AGN. Other features visible here are described in greater detail in Ouintana et al. (1995, 2000).

Figure 3 shows the redshift distribution for the 6dFGS+FLASH galaxy catalog and the radio galaxy subsample. The two outstanding peaks in the observed redshift distribution are the Hydra cluster at $\sim 4000 \mathrm{~km} \mathrm{~s}^{-1}$ and the $\mathrm{SSC}$ at $\sim 14400 \mathrm{~km} \mathrm{~s}^{-1}$. The large transverse filament at $16000 \mathrm{~km} \mathrm{~s}^{-1}$ seen in the wedge plots of Figure 2] also produces a wide peak in the redshift distribution histogram.

Both figures 2 and 3 show that, at first glance, radio-detected galaxies trace fairly well the underlying galaxy distribution: radio-detected galaxies are mostly found in the dense regions and less likely in the voids. However, Figure 2 indicates that the SSC-CR seems is devoid of strong radio sources, which cluster more in the A3528 cluster complex and its surroundings.

Since our radio galaxy classification is based upon a luminosity cut, it therefore creates a strong distance segregation, caused by Malmquist bias, where only luminous radio galaxies are present at larger distances. But since we restrict our analysis to a subsample doubly complete in volume and radio luminosity, Malmquist bias is no longer a concern.

\section{Radio emission in the A3558 and A3528 complexes}

According to our definition of the cluster complexes (Sect. 2.4), there are 1819 galaxies, among which 88 SBGs and 23 AGN, in the remaining FLASH+6dFGS area (outside both cluster complexes and with the velocity cut of eq. [2]). This sample will be used as a comparison sample and hereafter referred to as our reference sample. 


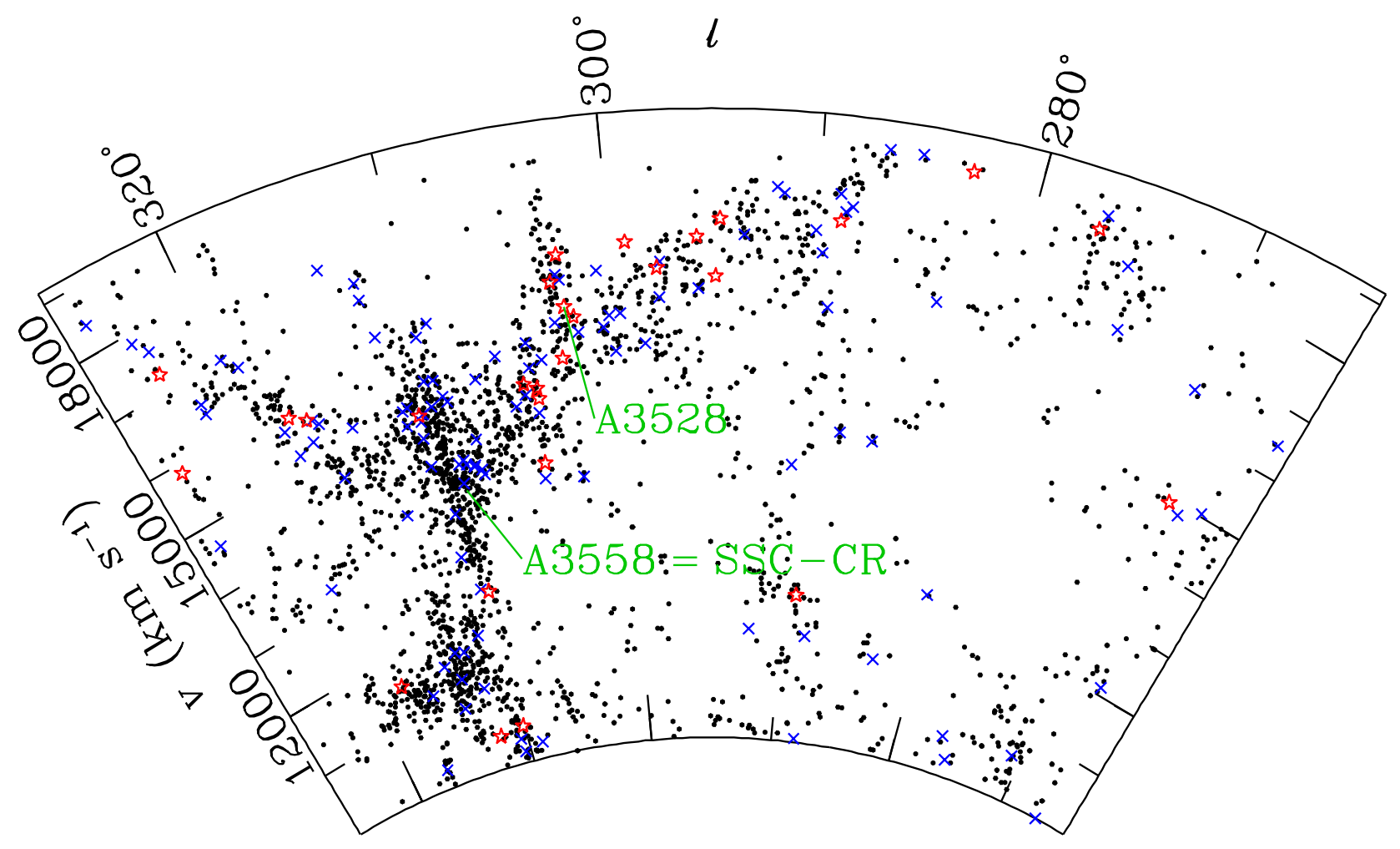

Fig. 2. Wedge plot in galactic longitude and heliocentric velocity of the FLASH survey area showing both FLASH and 6dFGS galaxies (black dots) and cross-identified radio-detected galaxies: AGN are shown as red open stars and SBGs as open blue crosses. The SSC is the Y-shaped large-scale structure in the left part of the plot. The two cluster complexes studied here are shown.

The fraction of radio-detected galaxies (SBG+AGN) are $4.7 \%, 8.3 \%$ and $6.1 \%$ in the A3558 complex, the A3528 complex, and the reference sample. Binomial statistics indicate that the lower (higher) fraction of radio galaxies $\left(\log L_{1.4 \mathrm{GHz}} \geq\right.$ 22.41) in the A3558 (A3528) complex relative to the reference sample is not significant.

Considering separately SBGs and AGN (with eq. [4]), we find 20 SBGs and no AGN in the A3558 cluster complex, whereas there are $5 \mathrm{AGN}$ and 5 SBGs in the A3528 cluster complex. Binomial statistics indicate that the absence of AGN among 20 radio-detected galaxies in the A3558 complex would occur by chance only $1.0 \%$ of the time, given the observed fraction $(23 / 111=21 \%)$ of AGN in the reference sample. On the contrary, the A3528 complex seems to harbor too many AGN relative to the reference sample, as there is only a $3.8 \%$ chance of obtaining at least 5 AGN among 10 radio galaxies given the fraction of radio-detected galaxies that are AGN in the reference sample.

The top plot of Figure 4 shows the cumulative radio luminosity function (RLF) of the A3558 and A3528 complexes. Of course, one can argue that it is dangerous to compare luminosities of samples at different distances, even with the same magnitude limit, as the more distant sample will lead to more luminous galaxies. However, A3528 is only $10 \%$ more distant than A3558, which should lead to luminosities typically $20 \%$ larger, i.e. a 0.08 increase in $\log L_{1.4 \mathrm{GHz}}$.

The plot clearly indicates a lack of high radio luminosity galaxies in the A3558 complex, which is marginally significant
(94\% confidence with a Kolmogorov-Smirnov - KS - test, 95\% with a Wilcoxon rank sum test).

However, for low radio luminosities, the radio galaxy distribution in the A3558 cluster complex resembles that in the reference sample, while the two distributions depart from one another only at intermediate and high radio luminosity. On the other hand, the A3528 complex shows an RLF that appears shifted to higher radio luminosities in comparison with the RLF of the reference field, but the difference is only marginally significant (91\% confidence with a KS test).

Splitting radio-detected galaxies into two classes, above and below an arbitrary limit of $\mathcal{R}_{K}=0.8$ (the median is 0.7 ), we find only 4 radio-loud galaxies out of 20 in the A3558 cluster complex (20\%) and 5 in the A3528 complex (50\%). In the reference sample, the fraction of radio-loud galaxies is 0.43 . Binomial statistics indicate that the probability that the A3558 complex would have as few as 4 galaxies with $\mathcal{R}_{K}>0.8$ amongst a total of 20 radio-detected galaxies is $3 \%$ : the lack of radio-loud galaxies in the A3558 complex is statistically significant. This conclusion depends little on our cut in radio loudness in the range $0.6<\mathcal{R}_{K}<0.9$.

The bottom plot of Figure 4illustrates this lack of radio-loud galaxies in the A3558 complex: the distribution of $\mathcal{R}_{K}$ in the A3558 complex is shifted to values $\approx 2 \times$ lower than for the reference field, and a KS test gives a $4 \%$ probability of a greater difference by chance (only $1 \%$ with a Wilcoxon rank sum test). On the other hand, the distribution of radio-loudness in the A3528 

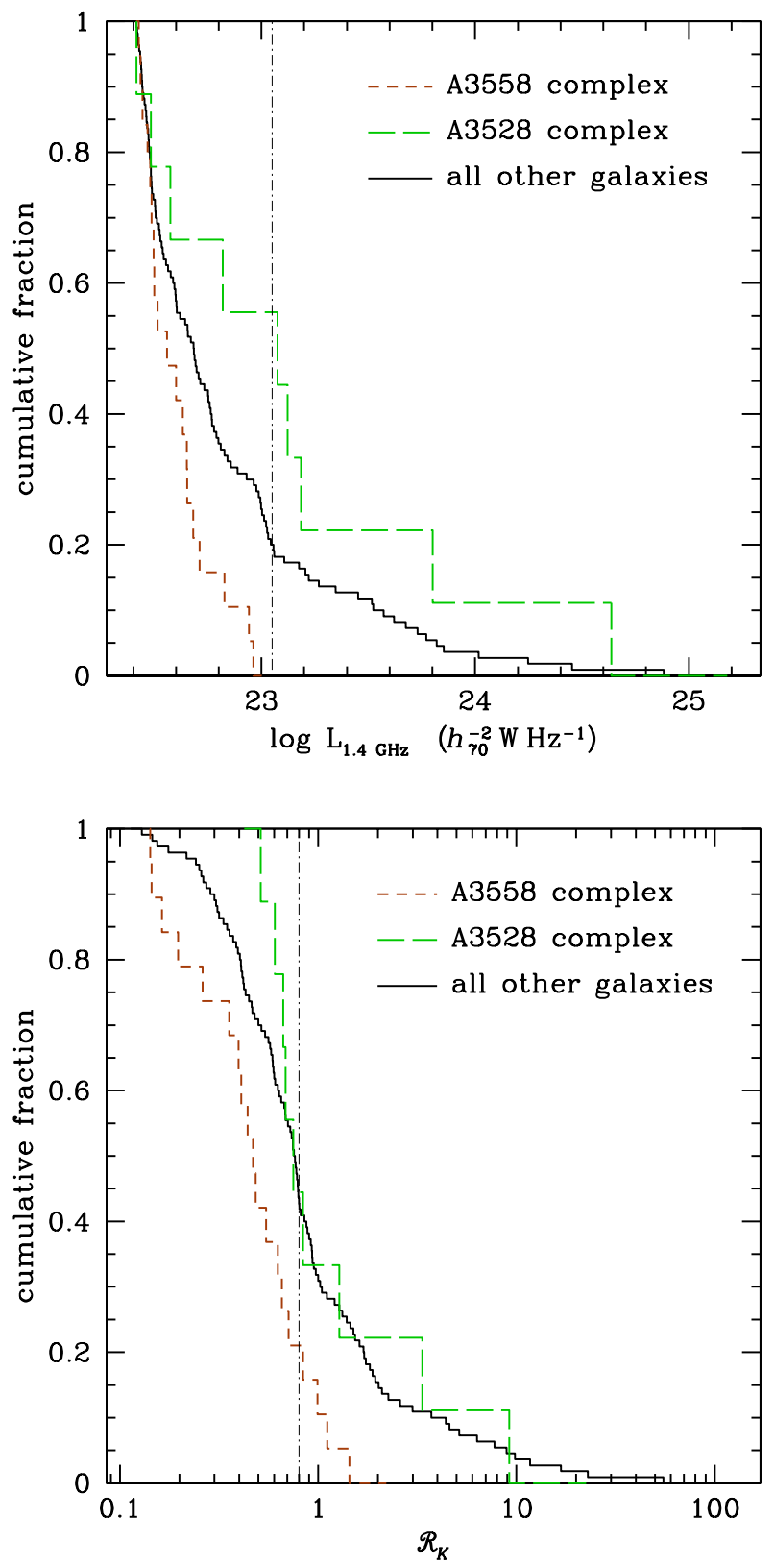

Fig. 4. Normalized cumulative distributions of radio luminosity (top) and radio loudness (bottom) for three regions: the A3558 complex (brown short-dash histogram), the A3528 complex (green long-dash histogram) and the reference sample (black solid histogram). The vertical lines denote the transition from SBGs to AGN (top plot) and our ad hoc separation of radio loudness (bottom plot).

complex is shifted to higher values, but because of small number statistics this offset is not significant.

To summarize, the two cluster complexes show different trends in their distribution of radio luminosities and radio loudness relative to the reference sample. the A3528 complex galaxies are marginally more radio-luminous than the galaxies in the reference sample, while the A3558 complex galaxies are marginally less radio-luminous and significantly less radio-loud.

These distributions tell us that the special dynamical state of the very dense core of the Shapley supercluster has a quan- tifiable impact on its radio galaxy population that significantly reduces the radio activity. However, these radio luminosity and radio-loudness distributions do not allow us to quantify what is the impact of their respective environments. We therefore investigate in the next section how these variables are affected by galaxy density, at the supercluster scale as well as inside clusters within the cluster complexes to determine what environmental processes might be at work.

\section{Modulation of radio emission with the density of the environment}

\subsection{Estimation of the density of the environment of radio-detected galaxies}

As seen in Figure 3, the fraction of radio-detected galaxies is independent of redshift. This means that we can estimate the density of the environment using the optical sample.

Given the discrete nature of the galaxy positions, we choose to measure a continuous density. We thus define a continuous density in redshift space, by smoothing the discrete density in redshift space with a Gaussian kernel of scale $\sigma_{r z}$ (c.f. Tully 1988; Monaco et al. 1994), using:

$\rho_{r z, j}\left[h^{3} \mathrm{Mpc}^{-3}\right]=\frac{1}{\left(2 \pi \sigma_{r z}^{2}\right)^{3 / 2}} \sum_{i \neq j} \exp \left(\frac{-r_{i j}^{2}}{2 \sigma_{r z}^{2}}\right)$,

where subscripts $j$ and $i$ respectively represent radio and optical galaxies and where

$$
\begin{aligned}
r_{i j} & =\sqrt{d_{p}^{2}\left(z_{1}\right)+d_{p}^{2}\left(z_{2}\right)-2 d_{p}\left(z_{1}\right) d_{p}\left(z_{2}\right) \cos \theta_{i j}} \\
& \simeq \frac{c}{H_{0}} \sqrt{z_{i j}^{2}+\left(\theta_{i j}\langle z\rangle\right)^{2}},
\end{aligned}
$$

is the separation of two galaxies in redshift space 6 , with $\theta_{i j}$ their angular separation and $\langle v\rangle$ their mean radial velocity, and where $d_{p}(z)$ is the proper distance.

Of course, such an estimator will be subject to edge effects (the smoothed density will decrease near the survey edges) and to the radial selection function inside the area. To correct for this, we generated 25 random datasets, each with as many galaxies as in our observed sample, in the exact same survey geometry and with the same velocity selection function, approximated with a 4th order polynomial fit to the velocity distribution, as shown in Figure 3 . Note that the mean local densities of the 25 random catalogs obtained with equation (6) will be $1 / 25$ th of the local densities of a single random catalog with 25 times as many galaxies. For each smoothing scale, we calculate the Gaussiansmoothed density and divide it by the mean Gaussian-smoothed density of our random catalogs.

In redshift space, the 3D density is biased by redshift distortions, i.e. incorrect radial positions of cluster galaxies due to fingers of God. We therefore also derive a continuous surface density of galaxies by Gaussian smoothing the discrete surface density, as:

$\rho_{\theta, j}\left[h^{2} \mathrm{Mpc}^{-2}\right]=\frac{1}{2 \pi \sigma_{\theta}^{2}} \sum_{i \neq j} \exp \left(\frac{-\theta_{i j}^{2}}{2 \sigma_{\theta}^{2}}\right)$,

\footnotetext{
${ }^{6}$ We actually use the cosmological formula, only valid for a flat Universe (first equality of eq. [7), but at the small redshift of our sample $(z<0.06)$, cosmological corrections are typically less than $4 \%$.
} 
again dividing by the mean surface density of the 25 random catalogs. Even though both approaches suffer biases, the combination of the two will allow us to check for consistencies in the trends.

We calculated densities for a wide range of smoothing scales, each separated by a factor of $2: \sigma=0.625,1.25,2.5,5,10$, and $20 h_{70}^{-1} \mathrm{Mpc} 7$ Of course, large scales suffer from a smaller range of smoothed densities. Small scales will suffer from the lack of close neighbors (in both the real and random catalogs). In addition, the surveys are not complete in crowded areas because of fiber avoidance and we therefore underestimate the density in the denser regions.

We illustrate our procedure by showing, in the top panels of Figure 5, the modulation of near-infrared luminosities with density, for two smoothing scales. One can easily notice a trend of luminosity segregation: the more luminous galaxies prefer the dense environments, especially at a smoothing scale of $2.5 \mathrm{Mpc}$, which is roughly the scale of clusters. Spearman tests indicate a rank correlation of -0.32 and -0.22 at 2.5 and $10 \mathrm{Mpc}$, respectively, which are over $99.5 \%$ significant. At a smoothing scale of $1.25 \mathrm{Mpc}, \mathrm{K}$-band luminosity is significantly anti-correlated with density, regardless of whether density is measured in projected or angular space. But at smoothing scales $\geq 2.5 \mathrm{Mpc}, L_{K}$ is not significantly anti-correlated with projected density.

\subsection{Radio luminosity - density relation}

The middle panels of Figure 5 show that the largest radio luminosities appear to prefer intermediate densities, near the median. However, the distribution of radio luminosities of galaxies at intermediate densities is not significantly different from the analogous distribution at extreme (high and low) densities. And, for all smoothing scales, there are no significant correlations of radio luminosity with both redshift space density and projected density.

\subsection{Radio loudness - density relation}

The correlation between a galaxy radio luminosity and the density of its environment might hide a correlation between radio luminosity and mass (as both are extensive variables) on one hand, and mass and environmental density on the other hand (as expected in models of galaxy formation where the more massive galaxies are more clustered). In other words, since more luminous galaxies are usually more clustered, and should have undergone several major mergers, they might also be prone to radio activity. We therefore correlate the radio loudness (eq. [5]) with the density of the galaxy environment.

As seen in the bottom right panel of Figure 5 , for smoothing scale $10 \mathrm{Mpc}$, radio loudness appears to be anti-correlated with the density of the environment: the low loudness galaxies are virtually all at high densities. This is quantified in Figure 6 which indicates highly significant anti-correlations of radio loudness with the density of the environment, measured on scales greater than $2 \mathrm{Mpc}$. These significant anti-correlations occur in both projected and redshift spaces, which gives us confidence in these observed trends. For example, at a smoothing scale $\sigma=10 \mathrm{Mpc}$, both $\rho_{\theta}$ and $\rho_{r z}$ are negatively correlated $(r \sim-0.17$ and $r \sim$

\footnotetext{
${ }^{7}$ For the density in projected space, we used for $\sigma_{\theta}$ in equation 8 angles of $\sigma /(200 \mathrm{Mpc})$, corresponding to the angular sizes of the redshift space smoothing scales at the distance of the SSC. In what follows, we will replace $\sigma_{\theta}$ by the equivalent scale in Mpc.
}

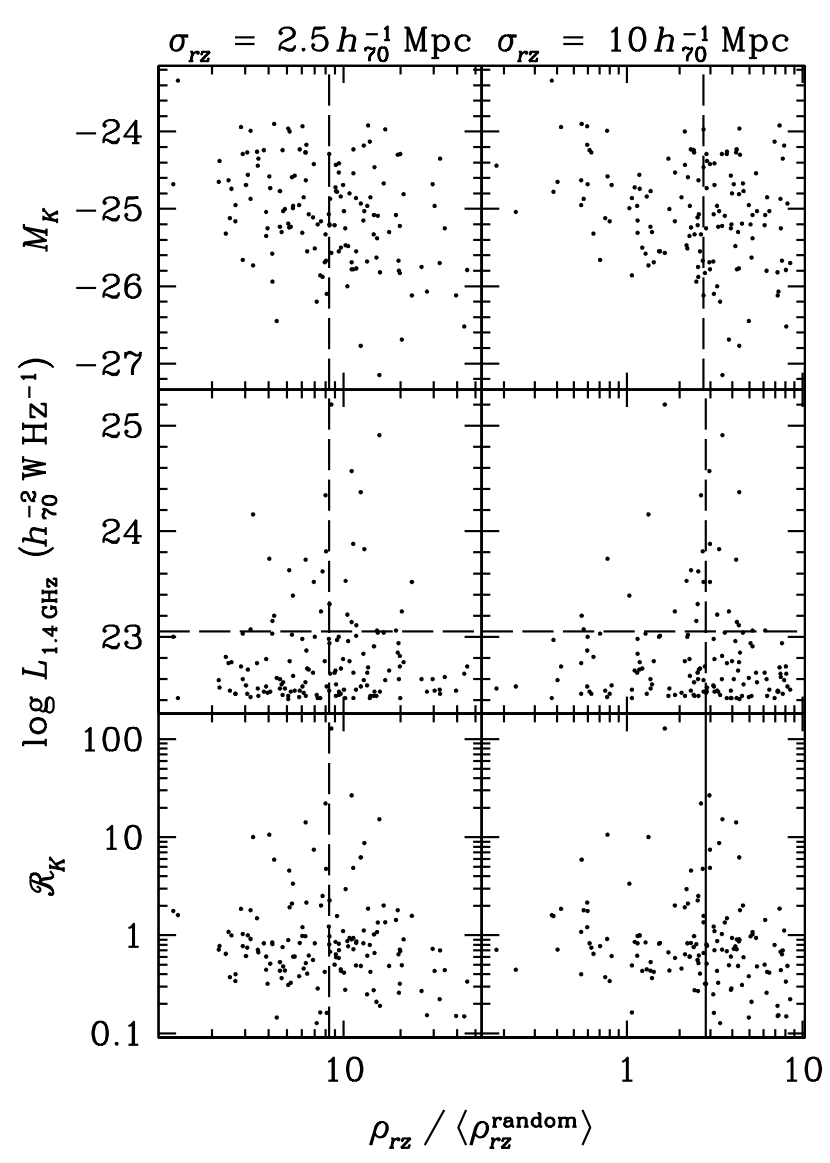

Fig. 5. Gaussian-smoothed density in redshift-space corrected for edge effects and the radial selection function versus $K$-band luminosity (top plot), radio luminosity (middle plot) and radio loudness (bottom plot), for two different smoothing scales. The dashed vertical and dashed horizontal lines represent the limit between high and low density environment and the separation between SBGs and AGN, respectively (see text).

$-0.21)$ with $\mathcal{R}_{K}$ with a high significance $(98 \%$ and $99.4 \%$, respectively).

We also used Kolmogorov-Smirnov (KS) tests to compare the distributions of densities for AGN vs. SBGs, as well as the distributions of radio luminosities, loudness (and NIR luminosities) for galaxies in high vs. low density environments. No significant trends were found at any smoothing scale in both analyses of projected and redshift space densities. This shows that the KS test is not as an efficient estimator of the environmental effects as is the rank correlation with density.

\subsection{Cutting out the cluster complexes}

To better understand the anti-correlation of radio loudness with density, we performed the same analysis as in Sect 4.3 on the FLASH area, but with parts of the SSC cut out from the sample. We calculate densities only for the radio-detected galaxies outside the A3558 cluster complex, whose projected radius is now a free parameter. Figure 6 shows how the significance of correlation $(P \rightarrow 0)$ or anti-correlation $(P \rightarrow 1)$ of radio loudness with redshift space density varies with the smoothing scale, for 


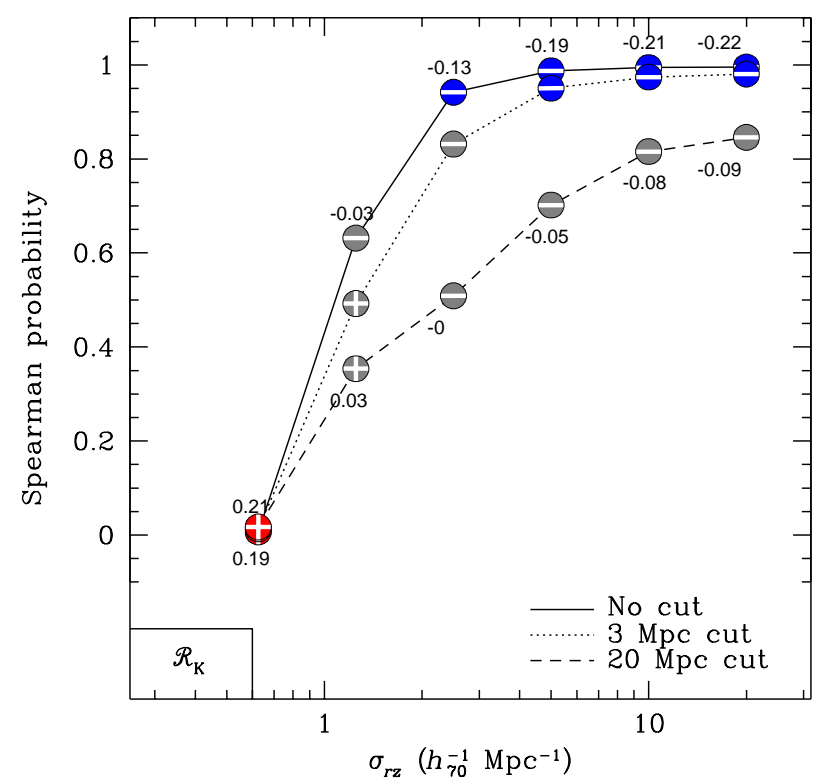

Fig. 6. Probability of statistical significance of Spearman rank correlation of radio loudness with Gaussian-smoothed density in redshift space (normalized with the mean density derived from the random catalogs) versus the smoothing scale. Dotted and dashed curves represent the analysis with the central portion of the SSC cut out to a radius of respectively $3 \mathrm{Mpc}$ and $20 \mathrm{Mpc}$, and limited to $\pm 3 \sigma_{v}$ (A3558). Red positive signs highlight positive correlation coefficient and blue negative signs the negative ones. The Spearman probability is defined as $P_{\text {Spearman }}=$ $\left(P_{\text {chance }}-1 / 2\right) \operatorname{sgn}(r)+1 / 2$. The values of the rank correlation coefficient $r$ are given for the two extreme cases.

different choices of the projected radius of the SSC-CR we cut from our sample.

At large cut sizes, the anti-correlation between radio loudness and local density at large scales disappears when removing the central region of the Shapley supercluster from the statistical analysis. The change in the statistical trend becomes visible at a cut size of $\sim 4 \mathrm{Mpc}$. As the projected radius of the removed area increases, the anti-correlation vanishes. When reaching a cut size of $\sim 6 \mathrm{Mpc}$, all the Spearman probabilities drop below $90 \%$. However, if we perform a similar analysis by removing a $20 \mathrm{Mpc}$ shell centered on A3528, we do not see any loss of anti-correlation at large scales, which means that this area is not responsible for the anti-correlation.

Since the SSC-CR seems to be the main area that disfavors the presence of radio-loud galaxies, we now compare the A3558 complex with its surroundings. As can be seen in Figure 7 there is a strong anti-correlation between radio loudness and the $\sigma_{r z}=10 \mathrm{Mpc}$ smoothed density among just the SSC-CR members. The Spearman rank-correlation coefficient is $r=-0.41$, which indicates a $97 \%$ level confidence for the negative trend. Outside the SSC-CR, radio loudness is uncorrelated with density. As noted before, there are virtually no radio-loud galaxies in the SSC-CR. Moreover, there seems to be an excess of galaxies of low radio loudness in the densest environments at $\sigma_{r z}=10 \mathrm{Mpc}$.

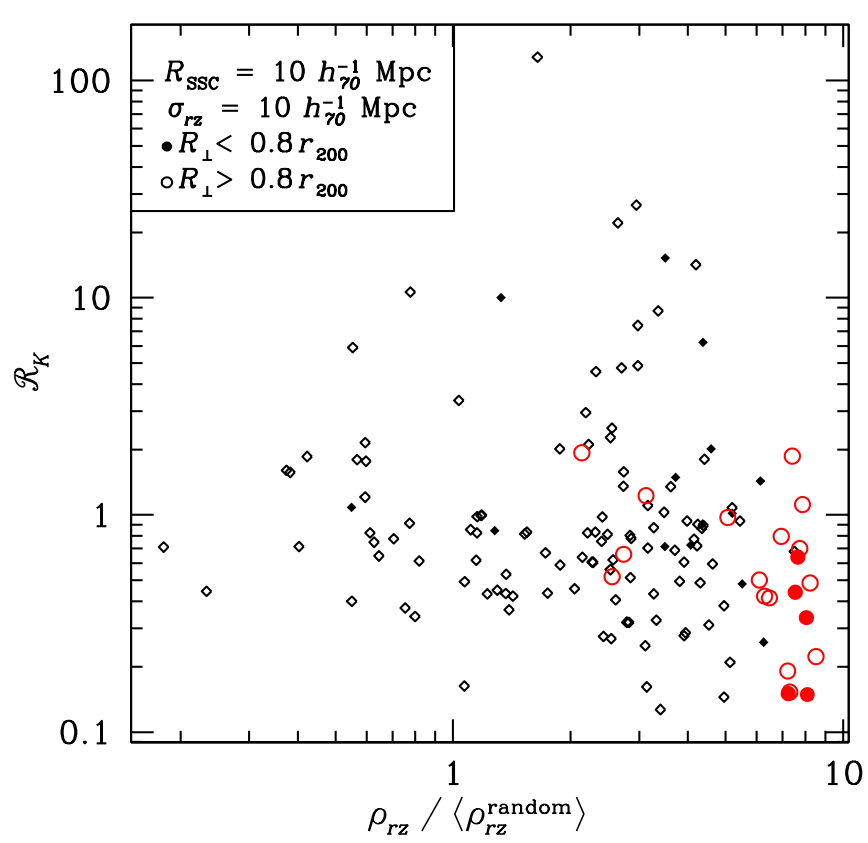

Fig. 7. Radio loudness ( $K$-band normalized) vs. density (smoothed on scale $\sigma=10 \mathrm{Mpc}$ ), separating the regions within (big red circles) and outside (small black diamonds) the SSCCR. Filled symbols show radio-detected galaxies that are less than $0.8 r_{200}$ of a galaxy cluster center whereas open symbols are the ones located at more than $0.8 r_{200}$.

\section{Modulation with the relative clustercentric distance}

We also identify radio-detected galaxies within and outside of clusters in the SSC-CR. For each radio galaxy, we search clusters whose redshift matches the galaxy to within 3 cluster velocity dispersions (measured with NED, which although inhomogeneous is more complete than 6dFGS). The smallest projected clustercentric distance, normalized to the cluster virial radius, $r_{200}$, where the mean total mass density of the cluster equals 200 times the critical density of the Universe, determines which cluster the radio galaxy is closest to. This avoids assigning more than one cluster to each radio galaxy. We estimated $r_{200}$ from the cluster velocity dispersions according to $r_{200}=\sigma_{v} / 436 \mathrm{~km} \mathrm{~s}^{-1}$, appropriate for the NFW model of concentration $c=5$, as derived in equation A.11. We then separate the galaxies within the cluster inner regions, defined as $0.8 r_{200}$, from the galaxies in the outer regions (extending to far beyond the cluster virial radius).

Figure 7 shows that outside of clusters, the distribution of radio loudness values is the same for regions within and outside the SSC-CR. However, Figure 7 also indicates that, within the SSC$C R$, the cluster radio-detected galaxies have lower (typically by a factor 3) radio loudness than galaxies in clusters outside the $S S C-C R$.

Table 1 provides KS test probabilities that the differences in the distributions of various parameters within and outside the SSC-CR are caused by chance, for radio-detected galaxies outside of clusters (first line) or for those within clusters (second and third lines, the latter omitting the central brightest cluster galaxy, hereafter BCG). The KS test indicates that the 3 times lower values of $\mathcal{R}_{K}$ of the cluster radio-detected galaxies in the 
SSC-CR relative to their counterparts outside of the SSC-CR is very highly significant: there is a $99.7 \%$ probability that the difference in distributions of radio loudness is not caused by chance.

Table 1. Significance (with KS test) of same distributions within and outside of the SSC-CR

\begin{tabular}{c|cccc}
\hline \hline Galaxy position & $L_{1.4 \mathrm{GHz}}$ & \multicolumn{1}{c}{$\mathcal{R}_{K}$} & $M_{K}$ & $B-K$ \\
\hline$R>0.8 r_{200}$ & 0.14 & 0.58 & 0.55 & 0.95 \\
$R<0.8 r_{200}$ & 0.10 & 0.003 & 0.05 & 0.77 \\
$0.04 r_{200}<R<0.8 r_{200}$ & 0.32 & 0.01 & 0.08 & 0.97 \\
\hline
\end{tabular}

Note: The galaxy position is relative to the center of the nearest cluster.

Now, one could argue that the depressed loudness of radiodetected galaxies within clusters inside the SSC-CR may signify an intrinsic anti-correlation between radio loudness and NIR luminosity coupled with a correlation between NIR luminosity and density (luminosity segregation). However, as one can see in Table 11 while the differences in the distributions of $K$-band luminosities between cluster radio-detected galaxies within and outside of the SSC-CR are significant, they are not as significant as the differences in the distribution of radio-loudness values, even if the BCG is removed.

Since there are no highly radio-luminous galaxies in the SSC-CR, which we interpret as an absence of AGN, the depressed values of $\mathcal{R}_{K}$ for the radio-detected galaxies within SSCCR clusters may be an effect of enhanced morphological segregation within Shapley clusters: an enhancement of the early-tolate ratio among spiral galaxies would lead to reduced star formation efficiencies for spiral galaxies within clusters of the SSC. And yet, as seen in Table 1, the colors of radio-detected galaxies lying in clusters are not affected by their presence within the SSC-CR. This suggests that morphological segregation is not the cause of the significantly lower values of radio loudness for cluster galaxies in the SSC-CR relative to the cluster galaxies outside the supercluster core.

Hence, the physical parameter of radio-galaxies within clusters that is most affected by the SSC-CR environment is radio loudness. On the other hand, the distribution of radio-luminosity and radio loudness in the outer regions of clusters is unaffected by their presence within or out of the SSC-CR. Therefore, the anti-correlation of radio loudness with density of the environment is caused by the decreased radio loudness of radio-galaxies within SSC-CR clusters, relative to their counterparts outside of the $S S C-C R$.

The suppression of radio emission in the SSC cluster galaxies is even clearer in Figure 8, As seen in Table 2, inside the SSC$\mathrm{CR}$, radio loudness and relative position in the nearest cluster are indeed significantly correlated ( $r=0.50,99 \%$ confidence), while outside the SSC-CR there is no correlation between radio loudness and clustercentric position. Part of the loudness - radius correlation in the SSC-CR may be due to suppressed radio emission in the BCGs sitting in the cluster cores (Burns 1990). Since we used the Abell cluster centers given by Abell et al. (1989), which do not coincide with the positions of the BCGs, these central galaxies are visible in Figure 8. We visually checked with NED the positions of the BCGs and found all of them to lie within $0.04 r_{200}$.

The correlation between radio loudness and clustercentric radius remains significant ( $r=0.42,96 \%$ confidence) once the BCGs $\left(R / r_{200}<0.04\right.$, given the imprecise centers that we have

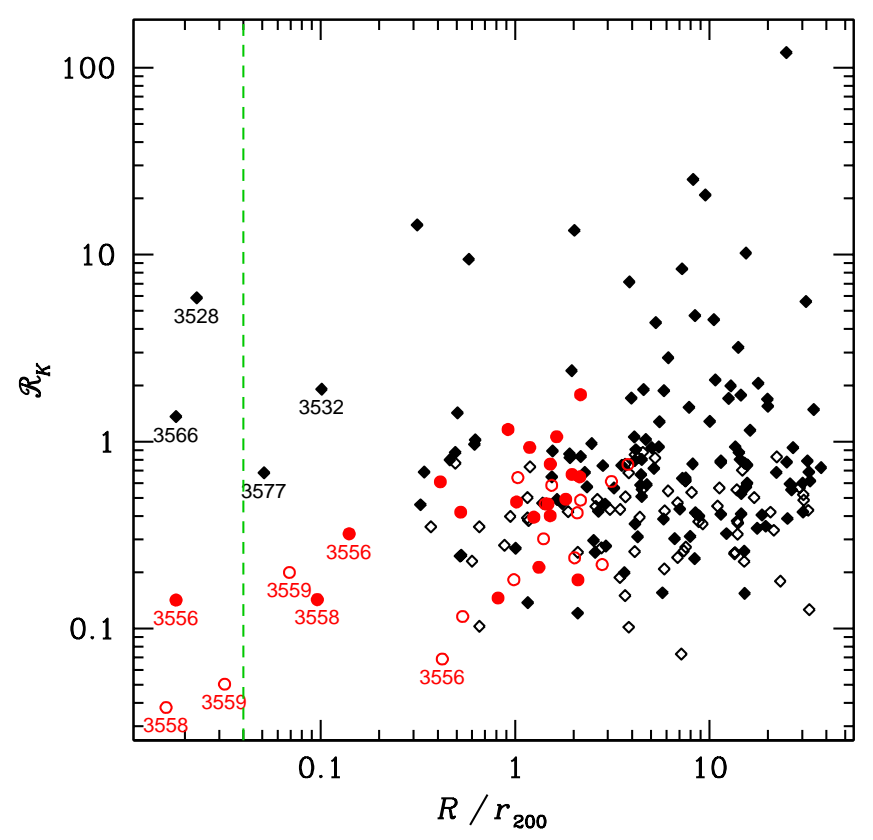

Fig. 8. Radio loudness ( $K$-band normalized) vs. projected distance to nearest cluster (in units of cluster virial radius). Red circles and black diamonds represent the galaxies within and outside the SSC-CR, respectively. The filled and open circles represent the galaxies with $\log L_{1.4 \mathrm{GHz}} \geq 22.41$ (complete radio luminosity sample in entire region) and $22.21 \leq \log L_{1.4 \mathrm{GHz}}<22.41$ (extension of sample, complete for the A3558 complex). The labels indicate the Abell cluster number of the galaxies with the lowest values of radio loudness. The galaxies left of the vertical line are brightest cluster galaxies, actually lying at the cluster center.

used) are removed. Figure 8 also shows an extension of the sample to lower radio luminosities, which is complete for the SSC$\mathrm{CR}$, but not for the full region outside the complex. The trends for low radio loudness of BCGs in the SSC-CR are confirmed, as is the same (weaker) trend for non BCGs within $0.3 r_{200}$ of the cluster center.

Table 2. Rank correlations with clustercentric radius and their significance (Spearman test)

\begin{tabular}{|c|c|c|c|c|c|}
\hline Zone & $R / r_{200}$ & $L_{1.4 \mathrm{GHz}}$ & $\mathcal{R}_{K}$ & $M_{K}$ & $B-K$ \\
\hline in & all & $-0.38(95)$ & $\begin{array}{ll}0.50 \quad(1) \\
\end{array}$ & $\begin{array}{ll}0.75 & (0)\end{array}$ & $-0.27(88)$ \\
\hline in & $>0.4$ & -0.35 (93) & 0.42 & 0.70 & -0.19 \\
\hline out & all & $-0.08 \quad(81)$ & 0.01 (46) & 0.17 & $0.04(32)$ \\
\hline out & $>0.4$ & -0.05 (69) & $0.04(32)$ & 0.15 & $0.07 \quad(22)$ \\
\hline
\end{tabular}

Notes: Column (1): within or outside SSC-CR; column (2): range of projected distances to the cluster center normalized to $r_{200}$; following columns: Spearman rank correlation coefficient, $r$, and associated probability $P_{\text {Spearman }}=\left(P_{\text {chance }}-1 / 2\right) \operatorname{sgn}(r)+1 / 2$ in percent in parentheses. The correlations all involve samples with $\log L_{1.4 \mathrm{GHz}} \geq 22.41$, so that all samples are complete in radio luminosity.

Table 2 also shows that in the SSC-CR, radio luminosity is marginally correlated with clustercentric position, but this correlation is absent outside the SSC-CR. On the other hand, the NIR luminosity is very strongly correlated with clustercentric radius, 
especially so in the SSC-CR, but also outside the cluster complex (i.e., NIR absolute magnitude is very strongly anti-correlated with clustercentric radius). The respectively strong and weak anti-correlations of NIR and radio luminosity with clustercentric radius inside the SSC-CR explain the positive correlation of radio loudness with clustercentric radius within the SSC-CR.

One could worry that the radio loudness - clustercentric radius correlation in the SSC-CR could be the consequence of the combination of 1) the strong NIR luminosity segregation, 2) the weak radio luminosity segregation, and 3 ) the radio luminosity limit of our sample. But since our sample is strictly limited in radio luminosity and virtually so in NIR luminosity (given the $K$-band limit of the 6dFGS sample and the volume limit that we imposed), selection effects should not affect radio luminosity significantly more than they affect NIR luminosity. Therefore, it is difficult to understand how selection effects could lead to a significant correlation of radio loudness with clustercentric radius in the SSC-CR. It therefore appears that the suppression of radio loudness is independent of the strong segregation in NIR luminosity, even when removing BCGs from our sample.

\section{Comparison with previous studies}

The distributions of radio luminosities in the A3558 and A3528 cluster complexes have previously been studied by Venturi et al. (2000), Miller (2005) and Venturi et al. (2001). Venturi et al. (2000) and Miller (2005) found that the cumulative radio luminosity function (RLF) of galaxies in the A3558 complex has a steeper bright-end slope than the respective reference samples of Ledlow \& Owen (1996) (E/S0 galaxies only) and Miller \& Owen (2001). This is in close qualitative agreement with the RLFs of the A3558 complex and the reference field that we have shown in the top plot of Figure 4. However, the radio luminosity where the SSC-CR RLF begins to depart from the reference one occurs at slightly different radio luminosities: $\log L_{1.4 \mathrm{GHz}} \lesssim 22.31$ for Venturi et al. (corrected to our cosmology), compared to $\log L_{1.4 \mathrm{GHz}}=22.6$ for Miller and 22.45 for us.

Venturi et al. also noted that the optically-defined radio loudness was uncorrelated with the density of the environment. Similarly, Miller did not see any spatial trends of opticallydefined radio loudness in the SSC-CR. Both results are in sharp contrast with the strong anti-correlation of NIR-defined radio loudness with the density of the environment and the strong correlation of radio loudness with clustercentric radius that we found for radio-detected galaxies in the A3558 complex. Note that while Venturi et al.'s sample is only very slightly deeper than ours in radio luminosity, Miller's sample is much deeper and had its AGN removed (by rejection of optically luminous galaxies).

For the A3528 complex, Venturi et al. (2001) found a normal (respectively lack by a factor 2) cumulative RLF of E/S0 galaxies in the A3528 complex if the their fraction of early-type galaxies is $100 \%(50 \%)$. But they saw no enhancement of the counts of radio-detected galaxies in contrast with the strong excess found in the optical. Although our statistics are poor, there is a marginal excess of radio luminous galaxies in the A3528 complex, as seen in the RLF of the top plot of Figure 4, and even in the wedge diagram of Figure 2. Our results can be reconciled with the results of Venturi et al. (2001), only by assuming that the shift is indeed not real as suggested by our marginal confidence level and that nearly all the galaxies in the Venturi et al. sample are early-type, which does not seem probable.
Gavazzi \& Jaffe (1986) found that optically-defined radio loudness was 3 times higher in clusters than outside clusters, but found no differences between their group, pair and isolated samples of radio-detected galaxies. In comparison, we find that radio loudness is enhanced in clusters by typically $60 \%$ outside of the SSC-CR, but decreased by a factor 3 inside the SSCCR. Best et al. (2007) find that AGN that are BCGs have the same distribution of radio luminosities as non-BCGs. We find (Table2) that radio luminosities of galaxies outside the SSC-CR are little affected by the position in the cluster, while the SSC$\mathrm{CR}$ galaxies have radio luminosities that are anti-correlated with clustercentric position, but that radio loudness is correlated with clustercentric position: hence $B C G$ s appear radio luminous, but given their very high NIR luminosities, their radio loudness is low.

There has been an accumulation of evidence over the last few years that cluster-cluster mergers at the heart of the SSC are responsible for the lack of radio-luminous galaxies in this region. The discovery by Brand et al. (2003) of a supercluster of radio galaxies is not in contradiction with this result, if their supercluster has not yet reached a stage of cluster-cluster merging, or if Brand et al. do not resolve the supercluster core where cluster merging may occur. Therefore, one has to be cautious when using radio-detected galaxies to trace large scale structures because the different dynamical stages of the collapse seem to play an important role in the presence of radio emission in galaxies.

\section{Physical processes at work in the core of the Shapley Supercluster}

There are several physical processes at work in a cluster-cluster merger that can affect the radio output of galaxies.

The suppression of radio emission in cluster galaxies within the SSC may be the result of cluster collisions, which heat up the central regions of clusters and destroy their cool cores. Indeed, Fabian et al. (1986) suggested that cooling flows fuel AGN and Burns (1990) found that the probability that a cluster $\mathrm{cD}$ galaxy hosted an AGN was 3 times greater (75\% vs. $25 \%)$ if the cluster has a cool core. Since A3558 has no cool core Sanderson et al. 2006), one expects the central galaxy of A3558 to have low radio loudness, as we observe. On the other hand, in X-rays, A3528 is made of two components, both with cool cores (Gastaldello et al. 2003), which is consistent with the high radio loudness of its cD. We are not aware of measurements of X-ray temperature profiles of the other clusters with central cDs: A3556, A3559, and A3566.

However, the reduced radio loudness in SSC-CR clusters is also present once the central regions are removed from the analysis (last line of Table 1), even though its significance is reduced. So, while cool cluster cores might be responsible for suppressing the radio loudness of central cluster galaxies in the SSC-CR, the suppression of radio emission also occurs outside the cluster centers.

The AGN mechanism is believed to rely on three ingredients: the presence of a massive black hole in the center of a galaxy, the supply of gas onto this black hole, and a mechanism to transport this gas to the central black hole. Starbursts also rely on the supply of gas onto molecular clouds.

Major mergers (of comparable mass galaxies) lead to violent relaxation that causes a substantial fraction of orbits to fall to the central regions and fuel the AGN (Roos 1981), as well as to compress the giant molecular clouds and induce a short but strong burst of star formation (Joseph \& Wright 1985). Rapid non- 
merging galaxy collisions (hereafter flybys) also have an important effect on galaxies: the tidal field at closest approach will generate barred instabilities (Gerin et al. 1990), which in turn will lead to efficient angular momentum exchange of stars and gas, some of which end up fueling the central AGN. Hence both galaxy mergers and rapid flyby collisions tend to boost the radio power of galaxies. Moreover, as we will discuss in Sect.7.3, the ram pressure that galaxies feel when orbiting within the hot intracluster gas can be altered within colliding clusters. One should note that these physical processes will affect the relative importance of the AGN/starburst activity relative to the mass of its host galaxy, which are measured by our radio-loudness $\mathcal{R}_{K}$.

We hereafter provide a thorough argumentation of the relative importance of several physical processes (major galaxy mergers, rapid galaxy flybys and ram pressure stripping of interstellar gas in galaxies by the intracluster gas) all believed to occur during a cluster-cluster merger. We discuss the impact of these processes on the AGN and SBGs, taking the A3558 cluster complex as an example.

\subsection{Increased or decreased direct major galaxy mergers within merging clusters?}

Much insight can be found in the simple case of two equal mass clusters merging in a head-on collision.

Consider first the situation at first pericenter, when the two clusters overlap. The rate at which a test galaxy merges with other galaxies of comparable mass is the sum of the major merger rate with galaxies from the first cluster and that with galaxies from the second cluster. Now, if the test galaxy originated in the first cluster, the rate of mergers with galaxies from the first cluster will be the same as when the two clusters were far apart.

In the limit where the cluster-cluster relative velocity is large, the test galaxy will suffer mergers with galaxies from the second cluster at a negligible rate, since the collisions will be too rapid to lead to galaxy mergers. In this regime, the test galaxy merges with other galaxies at the same rate as before the cluster-cluster encounter. For lower relative cluster-cluster velocities, the rate of mergers of the test galaxy with galaxies of the second cluster will no longer be negligible, hence the overall galaxy merger rate will be larger than it was before the cluster-cluster collision. Hence, there will be more occasions where the galaxy gas will be funneled down to the inner regions and fuel the central engine, thus triggering the AGN activity. And there will also be more occasions to tidally compress the molecular clouds and generate starbursts.

In appendix $\mathrm{B}$, we compute more precisely the rate of direct major galaxy mergers with galaxies of the other cluster at the moment of the first cluster overlap. For the SSC, the rms relative velocity of the clusters is small in comparison with their internal velocity dispersions. Indeed, as can be seen in Figure 9 the velocity dispersion is very low, $311 \mathrm{~km} \mathrm{~s}^{-1}$ for a projected apertures of $10 \mathrm{Mpc}$. However, the characteristic velocity relative to A 3558 will be $\sqrt{3}$ times larger, i.e. $538 \mathrm{~km} \mathrm{~s}^{-1}$. The rms internal velocity dispersion of the clusters within a projected distance of $10 \mathrm{Mpc}$ of A3558 is $786 \mathrm{~km} \mathrm{~s}^{-1}$. Then, according to Figure B.1, for luminous galaxies of velocity dispersion $\sigma_{g}=200 \mathrm{~km} \mathrm{~s}^{-1}$ inside rich clusters of velocity dispersion $\sigma=800 \mathrm{~km} \mathrm{~s}^{-1}$ (solid curve), with $V / \sigma=538 / 800=0.67$, we obtain an enhancement of the merger rate over $74 \%$. Hence, the total merger rate is almost twice that in the isolated cluster. For the merger rate

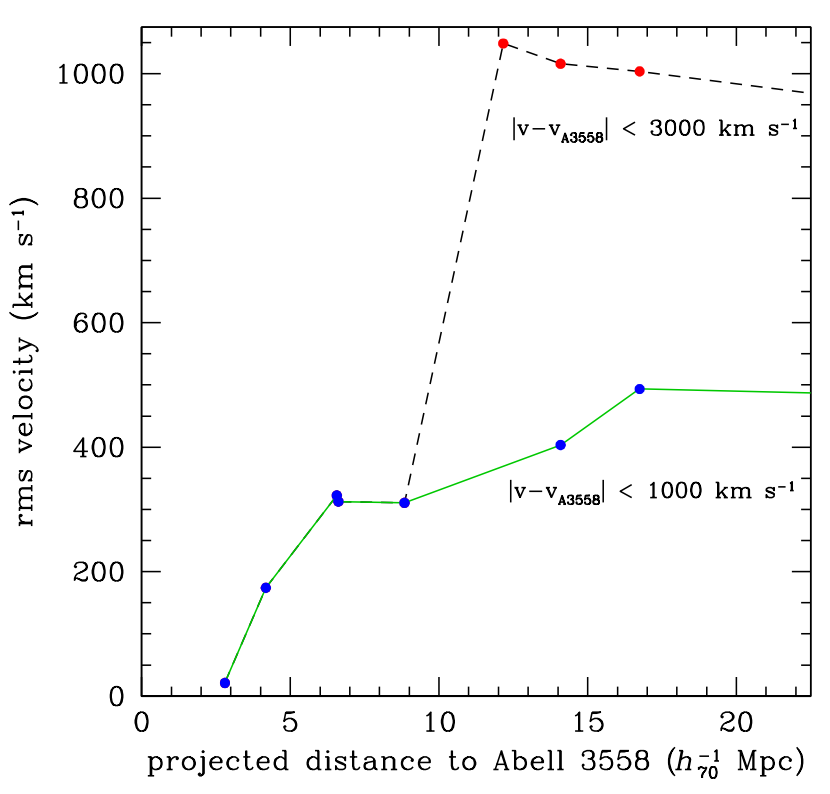

Fig. 9. Aperture velocity dispersion versus projected radius of the A3558 complex, using two velocity cuts to remove foreground/background interlopers. Each filled circle corresponds to the next closest cluster in projection to A3558, so the first symbol on the left is for 2 clusters, the next for 3 clusters, and so on.

of smaller galaxies, e.g. $\sigma_{g}=100 \mathrm{~km} \mathrm{~s}^{-1}$ (dashed curve), the enhancement is still over $67 \%$.

But the moment of cluster overlap is very short, hence may not be so relevant. After the first passage, the clusters will continue their course up to their apocenter, at which point the merger rate of galaxies of the first cluster with those of the second cluster will be near 0 , as the two clusters are far removed. The two clusters will then fall back onto one another for the final merger (see Barnes 1988 for the general case of merging galaxies).

The maximum separation of clusters having crossed one another is comparable to the maximum distance a galaxy can reach after having crossed right through the center of a cluster. This latter value has been estimated to be between 1 and 2.5 times the 100 overdensity radius (Mamon et al. 2004; Gill et al. 2005), which converts to roughly 1.3 to 3.2 times $r_{200}$. In fact, two equal mass clusters should feel a softer encounter than a tiny galaxy falling into a cluster, so that the maximum separation will be smaller (in relative terms). Now, the two clusters closest to A 3558 - A3556 and A3562 - lie at projected separations that correspond to 1.2 and 1.8 times $r_{200}$. Of course the separations in real (3D) space are greater or equal to these projected separations. Nevertheless, it is possible that A3556 and/or A3562 have already crossed through A3558.

In roughly one cluster crossing time, violent relaxation will lead to a relaxed merged cluster, and its galaxy major merger rate will scale as $1 / \sigma_{v}^{3}$ (Mamon 1992), which scales as $1 / M$, given the cosmological $M \propto \sigma_{v}^{3}$ relation applied to clusters. Hence, relative to the galaxy merger rate in each of the progenitor clusters, the total rate of galaxy mergers will rise from 1 at initial times to $\simeq 1.8$ at first pericenter, then back to $\sim 1$ at apocenter, and will tend to 0.5 once the two clusters have merged and violently relaxed (into a cluster twice their original mass). In other words, 
in an environment such as a supercluster, with ongoing cluster mergers, the galaxy merger rate in clusters will be boosted by a factor up to (during a short time at first pericenter) 1.8/0.5 $=3.6$ relative to galaxy merger rate in an isolated cluster of the same mass.

In the case of an off-axis cluster-cluster merging encounter, the boost in galaxy merger rates at first pericenter will be less than 3.6. But, since the galaxy merger rate in each of the two clusters will be at least greater than their original merger rate, the boost relative to the final merged cluster will be greater than $1 / 0.5=2$.

We now consider in more detail the clusters at some intermediate epoch after the first pericentric passage and before the final merger. It is well known that the tidal forces during an encounter convert the orbital energy of the colliding pair into internal motions that deform the systems. How will this deformation affect the rate of galaxy mergers? Some of the internal energy gained at the expense of orbital energy will be potential energy. To first order the deformation is the sum of a monopolar deformation that puffs up each cluster and higher order multipoles, for example possible tidal tails. These higher order terms have no incidence on the mean density. So the net effect of the gain in potential energy (the loss of potential energy in absolute value) will be a decrease in the number density of galaxies and a reduction of the rate of galaxy collisions. Moreover, part of the orbital energy will be converted into internal kinetic energy, leading to an increased velocity dispersion of the galaxy system, hence a lower fraction of collisions that will be slow enough to lead to mergers. So, increases in both the potential and kinetic components of the internal energy will combine to a decreased rate of galaxy mergers.

In other words, calling the potential and kinetic energies $W$ and $K$, using primes for the puffed up state and writing $R^{\prime}=R / x$ (where $x<1$ ), one has $W^{\prime} / W=x, \Delta K=-\Delta W=(1-x) W$ (from conservation of energy), $K=-2 W$ (virial theorem), and $K^{\prime} / K=\left(\sigma_{v}^{\prime} / \sigma_{v}\right)^{2}$. This leads to

$\frac{\sigma_{v}^{\prime}}{\sigma_{v}}=\sqrt{\frac{1+x}{2}}$.

As the merger rate can be written as $n k$, where $k \propto \sigma_{v}^{-3}$ and $n$ is the galaxy number density (Mamon 1992, and eq. [B.1]), the ratio of the galaxy merger rates between the puffed up cluster and the normal one is found to be (using eq. [9])

$\frac{n^{\prime} k^{\prime}}{n k}=2^{3 / 2} \frac{x^{3}}{(1+x)^{3 / 2}}$.

When one considers systems including a dissipative gas component, the global effect of the energy transfer is small on the non-dissipative component. While we are interested in the galaxy component of clusters, such simulations have not yet been performed in a realistic manner. However, the dark matter component, which should have very similar dynamics to the galaxy component, seems very little affected by the encounter. Indeed, as can be seen in the snapshots of merging clusters of Roettiger et al. (1993) and of merging galaxies of Di Matteo et al. (2007), the puffing up of the non-dissipative component appears to be of order of or less than $20 \%$, i.e. a factor $x \geq 0.8$. So to explain the factor two decrease in the fraction of radio-loud galaxies in the A3558 cluster complex (Sect. 3), using equation (10) one would therefore require $x=0.74$, i.e. a $35 \%$ increase in the cluster sizes, which appears to be inconsistent with the simulations quoted above.
To summarize, at first passage of the merging clusters, the rate of direct galaxy mergers is increased, while subsequently, before the final merger of the two clusters, the rate of galaxy mergers is decreased. Since the factor two reduction in the fraction of radio-loud galaxies in A3558 cluster complex requires an unrealistic $35 \%$ puff up in the clusters, we conclude that galaxy mergers cannot explain by themselves the reduced loudness in cluster radio-detected galaxies inside the SSC-CR.

\subsection{The effects of rapid flyby collisions}

Naturally, rapid flybys are less efficient individually than mergers in inducing star formation and AGN activity. But, in rich clusters, flybys are much more common than galaxy mergers. Mamon (2000) computed analytically both the rate of major mergers and the rate of flybys as a function of galaxy environment, mass and position in its group or cluster environment. His Figure 5 indicates a rate of flybys (defined such that they pump in an amount of energy at least one-third of the binding energy of the test galaxy) that, for rich clusters, is about 1 per galaxy per Hubble time, independent of galaxy mass. In comparison, ongoing galaxy merger rates in present-day rich clusters are almost always at rates less than 0.1 per galaxy, extrapolated to a Hubble time. So, in rich clusters, strong flybys are about 10 times more common than major mergers.

The rate of flybys is enhanced during the overlapping part of the cluster encounter. According to equation (19) of Mamon (2000), the rate of flybys varies as $1 / \sigma_{v}$, hence as $1 / M^{1 / 3}$. Just like for galaxy mergers, the total rate of flybys of galaxies from either cluster will be $\lesssim$ twice that in the individual progenitor clusters (without embarking into a calculation similar to that of appendix B], then will fall to below unity (see Sect.7.1) once the two clusters separate towards their apocenter, increases again at the final cluster merger, but then decreases after the final relaxation to reach a value of $2^{-1 / 3}$ the flyby rate in the individual clusters. The boost in the flyby rate relative to that in relaxed isolated clusters of the same mass will thus be $\lesssim 2^{4 / 3} \simeq 2.5$ at first pericenter. For off-axis cluster-cluster encounter, the flyby rate at first pericenter will still be greater than that in the individual progenitor clusters, hence the boost of the rate of flybys at pericenter, relative to that in isolated clusters of the same mass will be $2^{1 / 3} \simeq 1.3$.

Once the clusters pass their pericenter and puff up through their mutual tides (see Sect. 7.1), the rate of flyby encounters will be decreased. With the $1 / \sigma_{v}$ scaling of the rate of flybys, and using equation (9), we find that the ratio of the rate of flybys between the puffed clusters past pericenter and the individual clusters will be

$\frac{n^{\prime} k^{\prime}}{n k}=2^{1 / 2} \frac{x^{3}}{\sqrt{1+x}}$

Equation (11) then implies that to explain the factor of two decrease in the fraction of radio-loud galaxies in the A3558 cluster complex, one requires $x=0.78$, i.e. a $28 \%$ increase in the cluster sizes, which again appears to be inconsistent with the hydrodynamic simulations of Roettiger et al. (1993).

In summary, flybys are enhanced during the pericentric passage, and decreased afterwards, when the clusters puff up by their mutual tidal interaction. Yet, flybys cannot explain by themselves the factor two decrease in the fraction of radio loud galaxies in the core of the SSC. 


\subsection{Increased ram pressure stripping of galaxies in merging clusters}

We now argue that in merging clusters, as is the case in the inner regions of the SSC (Bardelli et al. 1998), ram pressure stripping of interstellar gas will be considerably more effective than in single clusters, which should lead to the starvation of the AGN activity as well as of star formation, and might thus explain the low levels of radio loudness for radio-detected galaxies within a virial radius of SSC-CR clusters, relative to those within a virial radius of clusters outside of the SSC-CR (Fig. 7 and Table 1 ).

When two clusters merge, their gaseous intracluster media, in contrast to their stellar and dark matter components, will not interpenetrate, but instead dissipate their orbital energies in a strong shock, caused by the adiabatic compression of the colliding gas clouds (Roettiger, Burns, \& Loken 1993). Therefore, a shock-heated interface should lie between the two clusters, reminiscent of what is seen in Stephan's Quintet (the HCG 79 compact group of galaxies, van der Hulst \& Rots 1981; Pietsch et al. 1997) and seen in all realistic hydrodynamical simulations of merging clusters (e.g. Roettiger et al. 1993). Behind the shock, the gas component should follow the same motion as the mean motion of the galaxies. The galaxies of the first cluster will see the relative speed of the gas change as they move from their initial parent cluster, through the shock to the other cluster. Since ram pressure is $P=\rho_{\text {gas }} v^{2}$, where $\rho_{\text {gas }}$ is the gas density and $v$ the velocity of the galaxy relative to the intracluster gas (Gunn \& Gott 1972), the efficiency of the ram pressure stripping will vary according to whether a galaxy is in it's initial parent cluster, in the shock at the cluster-cluster interface, or in the second cluster.

Consider two clusters of equal mass, with 1D velocity dispersion $\sigma$, merging along the $x$ axis at relative velocity $V$. At the moment it passes through the shock, the shocked gas that the galaxy encounters is 4 times denser than before the shock. Moreover, if the galaxy travels along the $x$ axis, the wind that the galaxy feels will typically be enhanced by $1+(V / 2)^{2} / \sigma^{2}$. Hence a galaxy traveling along the $x$ axis, passing through the shock interface, will feel a ram pressure $P^{\prime}$ enhanced by

$$
\frac{P^{\prime}}{P}=4+\left(\frac{V}{\sigma}\right)^{2} \text {. }
$$

Galaxies traveling in perpendicular directions will feel an enhanced ram pressure of a factor 4 , and their internal gas will be efficiently stripped by the ram pressure of the intracluster gas at the shock.

After the galaxy, traveling along the $x$ axis, passes through the shock, it will "see" the second cluster moving towards it at a velocity equal to the its velocity relative to the first cluster plus the cluster-cluster relative velocity. Hence, its interstellar gas will be ram pressure stripped by the intracluster gas of the other cluster much more efficiently, by a factor

$$
\frac{P^{\prime}}{P}=1+\left(\frac{V}{\sigma}\right)^{2} \text {. }
$$

Note that a galaxy moving within the first cluster along the $x$ axis, but in the same direction as the second cluster, will feel less ram pressure stripping during its initial infall and subsequent rebound. However, during its recoil 8 it will find itself in a similar

\footnotetext{
8 The recoil timescale, $\approx R_{\text {gal }} / \sigma_{v}$ should be shorter than the clustercluster merger time, $\approx R_{\text {cluster }} / V$, since the cluster radius $R_{\text {cluster }}$ is by definition larger than the radial position of the galaxy $R_{\text {gal }}$ and $V<$ $538 \mathrm{~km} \mathrm{~s}^{-1}<\sigma_{\mathrm{v}} \approx 800 \mathrm{~km} \mathrm{~s}^{-1}$.
}

situation as the galaxy that initially moves in the direction of the second cluster, and at this stage it will experience strong ram pressure stripping.

Galaxies traveling along axes perpendicular to the $x$ axis will feel the same ram pressure as if there had been no cluster-cluster merger. Therefore, within a merging cluster system, some of the galaxies that have penetrated the second cluster will suffer additional ram pressure stripping while others will feel about the same ram pressure as in their initial cluster.

Equations (12) and (13) suggest that for galaxies traveling along the cluster-cluster collision axis, the effect of the shock is more dramatic than the effect of the enhanced wind of the second cluster. Given the typical velocity dispersion of $800 \mathrm{~km} \mathrm{~s}^{-1}$ for clusters in the A3558 complex, with $V=538 \mathrm{~km} \mathrm{~s}^{-1}$, one finds that a galaxy moving along the cluster-cluster collision axis will see a ram pressure enhanced by a factor of 4.45 at the shock (eq. [12]) and by a factor 1.45 past the shock (eq. [13]). However, while the mild enhancement of ram pressure past the shock is long-lived, the thickness of the shock front is small in comparison with the cluster size (Roettiger et al. 1993), so that the galaxy will feel an enhanced density only for the short time it crosses through the shock. Therefore, it is not clear whether the short time during which the galaxy feels a ram pressure enhanced by a factor 4 will be sufficient for the interstellar gas to escape its host galaxy. It is beyond the scope of the present paper to predict which of the shock or enhanced wind from the 2nd cluster is the most efficient at decreasing the gas content of galaxies in merging clusters.

Note that the very low velocity difference, $21 \mathrm{~km} \mathrm{~s}^{-1}$, between A3558 and its nearest projected neighbor A3556 suggests that these two clusters have already passed through each other (unless their relative motion is perpendicular to the line of sight) and lost a substantial fraction of their initial orbital energy. The extra wind from the second cluster (A3556) will presently be of the same order as that from the first cluster (A3558). But before the two clusters lost their mutual orbital energy, the second wind must have been roughly twice as fast as the first one.

Moreover, ram pressure will strip the low density atomic gas clouds more efficiently than the dense cores of giant molecular clouds. The latter will be compressed by the ram pressure (Bekki \& Couch 2003). Therefore, if the galaxies feel a burst of ram pressure as they travel through the shock front, they should experience a burst of star formation and hence an enhancement of their radio emission. But once the atomic gas is removed, the subsequent star formation is starved. These issues are complex, probably require high resolution hydrodynamical simulations, and are beyond the scope of the present paper.

\subsection{Synthesis}

We have argued that, on one hand, environments such as the SSC which seem to harbor ongoing cluster-cluster merging collisions, should enhance the rates of direct galaxy-galaxy mergers (Sect. 7.1) and rapid flyby collisions (Sect. 7.2) at the first passage of the two clusters. But both rates should be decreased after the first pericentric passage and before the final cluster-cluster coalescence. However, neither galaxy mergers nor rapid flybys appear sufficient to explain the factor of two decrease in the fraction of radio loud galaxies in the A3558 complex at the core of the SSC.

On the other hand, we argued in Sect. 7.3 that both the enhanced density at the shock front of the cluster-cluster collision and the enhanced wind felt when galaxies pass through this shock ought to enhance the efficiency of the stripping of inter- 
stellar gas by the hot intracluster gas, which in turn will starve AGN and star formation.

Also, the enhanced ram pressure stripping should affect more than half the cluster galaxies that will have crossed the shock front (the orbital timescale within the cluster will be considerably shorter than the cluster-cluster merging time). On the other hand, only a few percent of cluster galaxies are expected to merge for the few Gyr duration of the cluster-cluster merger. Indeed, Figure 4 of Mamon (2000), shows that, at best, the current rate of galaxy mergers in clusters is of order of 0.14 per Hubble time, i.e. of order of 0.01 per Gyr. Hence, a reduction of a factor 2 to 3 in the galaxy merger rate after the first clustercluster pericentric passage will decrease the fraction of galaxies that merge during the last $3 \mathrm{Gyr}$ from roughly $3 \%$ to $1 \%$. If the bursts of star formation in clusters are mostly caused by infalling spirals from low velocity dispersion groups where the merger rate is high, the effect of the cluster-cluster collision on the rate of star formation should be negligible.

Moreover, while a decreased rate of galaxy mergers or flybys will explain the decreased fraction of radio-loud galaxies, only enhanced ram pressure stripping in merging clusters can explain that the few radio-detected non-BCG galaxies within SSC-CR clusters have values of radio loudness typically several times lower than radio-detected galaxies within clusters outside of the $S S C$-CR. Therefore, in an environment of merging clusters, it is likely that galaxies will have decreased radio loudness from the starvation of AGN and star formation activity caused by the enhanced ram pressure stripping of galaxies, unless the galaxies are in clusters that are just approaching for the first time, as appears to be the case for the A3528 cluster complex. The anticorrelation of radio loudness with the density of the environment that we find in the core of the Shapley supercluster suggests that clusters in this environment are going through a pericentric passage now, or have done so in the recent past.

The evolution of radio-detected galaxies should therefore be linked to the dynamical evolution of structures and to the merging of the different sub-structures, thus potentially explaining the apparent inconsistencies between various works (e.g. Owen et al. 1999 and Venturi et al. 2000). In addition, with less massive (and lower velocity dispersion) structures in the past, there would be more galaxy merging and less ram pressure stripping in cluster-cluster merging that would not quench star formation and the AGN phenomenon as well as it does nowadays. The radio-detected galaxies could therefore very well remain good tracers of large scale structures at high redshift (as found by Brand et al. 2003).

\section{Summary}

We merged two of the most recent optical/NIR catalogs (FLASH and 6dFGS) that cover a large superstructure (the Shapley Supercluster) as well as a fair number of clusters and voids. We then cross-identified our sample with the NVSS radio survey and restricted our study to a volume, flux and radio luminosity limited sample to limit biases in our studies. We retrieved 142 radio-detected galaxies, from which 28 were classified as AGN and 114 as SBGs.

To first order, radio-detected galaxies roughly trace the overall large scale structure of the area (Figs. 2] and 3). However, the fraction of galaxies with high $K$-band normalized radio-loudness in the A3558 cluster complex at the core of the SSC is half that in our reference sample (see Fig. 4). Moreover, we find that radioloudness is clearly anti-correlated with the large scale density of the environment (Fig. 6). This anti-correlation disappears when a large shell around the central region of the supercluster (SSC) is removed, indicating that this region is mostly responsible for this trend.

A detailed analysis indicates that this radio loudness / density anti-correlation is caused by the lower radio loudness of incluster $\left(r<0.8 r_{200}\right)$ radio-detected galaxies within the $10 \mathrm{Mpc}$ region centered on A3558 (the center of the SSC), in comparison with the in-cluster radio-detected galaxies outside the core of the SSC (see Fig. 7). The suppression of radio loudness in radio-detected galaxies appears most dramatic for BCGs in the SSC-CR (Fig. 8). But the suppression of radio loudness is also significant in non-BCG cluster radio galaxies, especially at $R<0.3 r_{200}$. While NIR luminosity segregation is extremely strong in the SSC-CR, it is also significant outside the SSC-CR (Table 2), so that the modulation by the SSC-CR of the distribution of radio loudness of in-cluster galaxies is at least as significant as the corresponding distribution of NIR luminosities (Table 1).

The radio luminosity distributions in the A3558 and A3528 cluster complexes show (Fig. 4) different behaviors that might highlight different dynamical states. The radio-detected galaxies in the A3558 complex have no highly radio-luminous galaxies and are significantly less radio-loud than in our reference sample, while those in the A3528 complex are significantly more radio-luminous and marginally more radio-loud.

In light of these results, we investigated possible causes for the suppression of radio loudness in cluster galaxies of the SSC core region, in the context of cluster-cluster mergers, which are thought to occur in this region. For the BCGs, a likely culprit is the disruption of the cool cluster core, which is believed to provide an efficient mechanism to fuel the AGN. For non-BCGs, using simple calculations, we show (Sect.7) that both direct major galaxy mergers and rapid collisions are enhanced at the moment of cluster overlap in comparison with isolated clusters of the same mass. However, after their first passage, the two colliding clusters will puff up (through their mutual tidal interaction), which leads to decreased rates of direct galaxy mergers and flybys. But we show that the cluster puff up is too small to explain the decreased fraction of radio-loud galaxies in the core of the SSC through galaxy mergers and rapid flybys. On the other hand, ram pressure is increased by galaxies passing through the shock front at the cluster-cluster interface, and also by the enhanced wind velocity they feel as they enter the second cluster. This enhancement of ram pressure will be most effective in the inner regions of clusters and should quench the AGN activity and starve the star formation, thus reducing the radio loudness of cluster galaxies in the SSC core, as is observed.

There are many perspectives to this work. The range of radio luminosities or radio loudness values where the suppression of radio emission occurs can be better assessed by studying a deeper radio sample of the SSC-CR, such as that of Miller (2005). The physical mechanisms at work can also be better constrained by distinguishing AGN from SBGs, using optical spectra or far IR diagnostics, and by incorporating galaxy morphologies to the analysis.

For example, one could test the effects of cluster-cluster mergers using the colors or spectra of galaxies: these will show a burst of star formation, whose age reflects the time of the first pericentric passage, since starburst timescales are much shorter than the dynamical timescales of structure merging. The variation of the star formation rate with time around pericenter should be skewed towards early times. Indeed, the passage of a cluster galaxy through the shock front and into the faster wind caused by the motion of the second cluster will abruptly starve the star for- 
mation that had just been enhanced by increased galaxy mergers at the beginning of the penetration of the two clusters.

Also, it would be interesting to see if the SSC-CR is unique or common, by performing similar analyses as done here on the core regions of other nearby superclusters. Finally, the details of ram pressure stripping, in particular the competition between the short but strong enhancement caused by passage of galaxies through the shock front generated by the cluster-cluster merger on one hand, and the faster wind felt after passage through the shock on the other hand, can be assessed by analyzing currently available hydrodynamical $\mathrm{N}$-body simulations of cluster mergers.

Acknowledgements. We thank Andrea Biviano, Avishai Dekel, Niruj Mohan, Nick Seymour, and especially Trevor Ponman for enlightening discussions. We are indebted to Gwenaël Boué for help with the Appendix, as well as to Andrea Biviano, Florence Durret, Gary Hill, Steve Rawlings for a critical reading of temporary versions of the manuscript. J.C.M. is grateful to Gary Hill for suggesting the topic and for hosting him at the University of Texas during the early stages of this work, which were supported, in part, by the Texas Advanced Research Program under Grant No. 009658-0710-1999. We also thank an anonymous referee for his numerous comments, which led to a significantly improved work. This publication makes use of data products from the Two Micron All Sky Survey, which is a joint project of the University of Massachusetts and the Infrared Processing and Analysis Center/California Institute of Technology, funded by the National Aeronautics and Space Administration and the National Science Foundation. The Digitized Sky Surveys were produced at the Space Telescope Science Institute under U.S. Government grant NAG W-2166. The images of these surveys are based on photographic data obtained using the Oschin Schmidt Telescope on Palomar Mountain and the UK Schmidt Telescope. This research has made use of the NASA/IPAC Extragalactic Database (NED) which is operated by the Jet Propulsion Laboratory, California Institute of Technology, under contract with the National Aeronautics and Space Administration. 


\section{Appendix A: Relation between virial radius and aperture velocity dispersion for an NFW model}

Given that the mass $M_{\mathrm{vir}}$ within the virial radius $r_{\mathrm{vir}}$ is $M_{\mathrm{vir}}=4 \pi / 3 \Delta \rho_{c} r_{\mathrm{vir}}^{3}$, where $\Delta$ is the mean density of the cluster within the virial radius, relative to the critical density $\rho_{c}=3 H^{2}(z) /(8 \pi G)$, one easily derives

$\sigma_{\mathrm{ap}}=\left(\frac{\Delta}{2}\right)^{1 / 2}\left(\frac{\sigma_{v}}{v_{\mathrm{vir}}}\right) H(z) r_{\mathrm{vir}}$,

where $v_{\text {vir }}=\left(G M_{\text {vir }} / r_{\text {vir }}\right)^{1 / 2}$ is the circular velocity at the virial radius.

Inspecting Figure 7 of Łokas \& Mamon (2001), one finds for an isotropic Navarro, Frenk, \& White (1996, hereafter NFW) model that the normalized aperture velocity dispersion $\widetilde{\sigma}_{v}=\sigma_{\mathrm{ap}} / v_{\mathrm{vir}} \simeq 0.7$. One can precisely compute $\sigma_{\text {ap }}$ for isotropic velocities with a simplified version of equation (47) of Łokas \& Mamon (2001) given by Mamon \& Łokas (2005):

$\sigma_{\text {ap }}^{2}(R)=\frac{4 \pi G}{M_{p}(R)}\left[\int_{0}^{\infty} r \rho(r) M(r) \mathrm{d} r-\int_{R}^{\infty} \frac{\left(r^{2}-R^{2}\right)^{3 / 2}}{r^{2}} \rho(r) M(r) \mathrm{d} r\right]$,

where $\rho, M$, and $M_{p}$ are the tracer mass density, total and projected mass, respectively (the Łokas \& Mamon formula involved a double integral instead of the two single ones in eq. [A.2]). Writing the density and mass of the NFW profile of virial radius $r_{\text {vir }}$ and concentration $c$ as (Mamon \& Łokas 2005

$$
\begin{aligned}
\rho(r) & =\frac{1}{g} \frac{M_{\mathrm{vir}}}{4 \pi r_{\mathrm{vir}}^{3}} \widetilde{\rho}, \\
M(r) & =M_{\mathrm{vir}} \frac{\widetilde{M}}{g},
\end{aligned}
$$

where

$$
\begin{aligned}
\widetilde{\rho} & =y^{-1}(1+y)^{-2}, \\
\widetilde{M}(y) & =\ln (y+1)-\frac{y}{y+1}, \\
y & =\frac{r}{a}=\frac{c r}{r_{\text {vir }}}, \\
g & =\widetilde{M}(c)=\ln (c+1)-\frac{c}{c+1},
\end{aligned}
$$

(where our definition of $g$ is the inverse of that of Łokas \& Mamon and where $a$ is the radius of density slope -2), equation (A.2) yields

$\widetilde{\sigma}_{v}^{2}\left(Y r_{\mathrm{vir}} / c\right)=\frac{c}{g} \frac{\int_{0}^{\infty} y \widetilde{\rho} \widetilde{M} d y-\int_{Y}^{\infty}\left(y^{2}-Y^{2}\right)^{3 / 2} \widetilde{\rho} \widetilde{M} d y / y^{2}}{C^{-1}(1 / Y) /\left|Y^{2}-1\right|^{1 / 2}+\ln (Y / 2)}$,

where $Y=R / a=c R / r_{\text {vir }}$ and

$C^{-1}(x)= \begin{cases}\cos ^{-1} x & (x<1), \\ \cosh ^{-1} x & (x>1),\end{cases}$

(see eq. [42] of Łokas \& Mamon 2001, note that the expression in brackets in eq. [43] of Łokas \& Mamon 2001 tends to $1-\ln 2$ for $Y=1$ ). Inserting equations (A.5), A.6 and A.8 into equation (A.9), yields a normalized aperture velocity dispersion at the virial radius $(Y=c)$

$\frac{\sigma_{\text {ap }}}{v_{\text {vir }}}=\widetilde{\sigma}_{v} \simeq \operatorname{dex}\left(-0.1539-0.2138 \log c+0.2358 \log ^{2} c-0.05357 \log ^{3} c+0.005515 \log ^{4} c\right)$,

which is accurate to better than $5 \%$ for $c>0.5$ and better than $0.12 \%$ for $c>1$. Thus, we find that $\widetilde{\sigma}_{v}$ reaches a minimum of 0.62 between $2<c<5$ and is equal to 0.66 for $c=10$.

With $H_{0}=70 \mathrm{~km} \mathrm{~s}^{-1} \mathrm{Mpc}^{-1}$ and $\Delta=200$, equations A.1 and A.10 lead to

$$
\left(\frac{r_{200}}{1 \mathrm{Mpc}}\right)=\frac{\sigma_{\mathrm{ap}}}{\sigma_{1}}, \quad\left(\frac{\sigma_{1}}{1 \mathrm{~km} \mathrm{~s}^{-1}}\right)=\left\{\begin{array}{ll}
433 & (c=4) \\
436 & (c=5) \\
463 & (c=10)
\end{array} .\right.
$$

Note that Carlberg, Yee, \& Ellingson (1997) assume $\sigma_{\text {ap }} / v_{\text {vir }}=1 / \sqrt{3}$, hence $\sigma_{\text {ap }=\sqrt{\Delta / 6} \mathrm{H}(\mathrm{z}) \mathrm{r}_{\mathrm{vir}}}$, which for $H_{0}=70 \mathrm{~km} \mathrm{~s}^{-1} \mathrm{Mpc}^{-1}$ and $\Delta=200$ yields $\sigma_{1}=404 \mathrm{~km} \mathrm{~s}^{-1}$. In other words, relative to a $c=5 \mathrm{NFW}$ cluster, Carlberg et al. overestimate the virial radius by $436 / 404-1=8 \%$. 


\section{Appendix B: Rate of direct galaxy mergers in fully overlapping merging clusters of equal mass}

The rate at which a galaxy suffers direct major mergers can be written

$\frac{d N}{d t}=n k_{1}$,

where $n$ is the number density of galaxies, while the merger rate $k_{1}$ (' 1 ' for single cluster) is (Mamon 1992)

$k_{1}=\langle v S(v)\rangle=\pi \int_{0}^{\infty} v f(v) p_{\text {crit }}^{2}(v) d v$,

with $S(v)=\pi p_{\text {crit }}^{2}(v)$ the merger cross-section and $f(v)$ the distribution of relative galaxy velocities, normalized such that $\int_{0}^{\infty} f(v) d v=1$. One typically assumes a Gaussian distribution of relative velocities, with standard deviation $2^{1 / 2} \sigma$, where $\sigma$ is the one-dimensional cluster velocity dispersion and the factor $2^{1 / 2}$ arises from the consideration of relative velocities, yielding

$f(v)=\frac{1}{2 \sqrt{\pi} \sigma^{3}} v^{2} \exp \left(-\frac{v^{2}}{4 \sigma^{2}}\right)$

which satisfies $\int_{0}^{\infty} f(v) d v=1$.

Equations (B.2) and (B.3) lead to

$k_{1}=\frac{1}{2 \sqrt{\pi} \sigma^{3}} \int_{0}^{\infty} v^{3} \exp \left(-\frac{v^{2}}{4 \sigma^{2}}\right) S(v) d v$.

The direct merger rate $k$ was computed by Mamon (1992, 2000) for the case of a linear critical impact parameter (Roos \& Norman 1979), yielding

$S(v)=\pi p_{0}^{2}\left(1-\frac{v}{v_{0}}\right)^{2}$

where $p_{0}$ is the maximum impact parameter that leads to a merger, while $v_{0}$ is the maximum velocity for head-on mergers.

We now consider the rate of direct galaxy mergers within a system of two equal mass merging clusters, at the moment when the two clusters overlap. We call $V$ the relative velocity of the two clusters at overlap. A galaxy initially in cluster 1, will suffer mergers with other galaxies of cluster 1 at a rate $k_{1}$ given by equation (B.4) and galaxies of cluster 2 at a rate $k_{2}$, with a total merger rate

$\frac{d N}{d t}=n_{1} k_{1}+n_{2} k_{2}=n\left(k_{1}+k_{2}\right)$.

The rate $k_{2}$ of direct major mergers that the test galaxy will suffer with galaxies of cluster 2 can be written as follows.

Consider a cylindrical coordinate system, whose axis joins the 2 clusters and call $v_{\|}$and $v_{\perp}$ the coordinates of a galaxy in this coordinate system in the frame of the 2nd cluster. In analogy with equation (B.3), one can then express the distribution of the velocities of the galaxies in the 2 nd cluster as

$f_{2}\left(v_{\|}, v_{\perp}\right)=\frac{1}{4 \sqrt{\pi}} \frac{v_{\perp}}{\sigma^{3}} \exp -\left(\frac{v_{\|}^{2}+v_{\perp}^{2}}{4 \sigma^{2}}\right)$,

which verifies $\int_{-\infty}^{+\infty} d v_{\|} \int_{0}^{\infty} f_{2}\left(v_{\|}, v_{\perp}\right) d v_{\perp}=1$, and where $\sigma$ is the velocity dispersion of the 2 nd cluster (equal to that of the first cluster). Noting that the velocity of a galaxy of the 2nd cluster relative to the test galaxy of the first cluster satisfies $v^{2}=\left(v_{\|}+V\right)^{2}+v_{\perp}^{2}$, the analog of equation (B.2) leads to

$$
\begin{aligned}
k_{2} & =\int_{-\infty}^{+\infty} d v_{\|} \int_{0}^{\infty} f_{2}\left(v_{\|}, v_{\perp}\right) v S(v) d v_{\perp} \\
& =\frac{\exp \left(V^{2} / \sigma^{2}\right)}{4 \sqrt{\pi} \sigma^{3}} \int_{-\infty}^{+\infty} \exp \left(\frac{v_{\|} V}{2 \sigma^{2}}\right) d v_{\|} \int_{\left|V+v_{\|}\right|}^{\infty} v^{2} S(v) \exp \left(-\frac{v^{2}}{4 \sigma^{2}}\right) d v \\
& =\frac{1}{4 \sqrt{\pi} \sigma^{3}} \exp \left(\frac{V^{2}}{\sigma^{2}}\right) \int_{0}^{\infty} v^{2} S(v) \exp \left(-\frac{v^{2}}{4 \sigma^{2}}\right) d v \int_{-V-v}^{-V+v} \exp \left(\frac{v_{\|} V}{2 \sigma^{2}}\right) d v_{\|} \\
& =\frac{1}{\sqrt{\pi} V \sigma} \exp \left(-\frac{V^{2}}{4 \sigma^{2}}\right) \int_{0}^{\infty} v^{2} \sinh \left(\frac{v V}{2 \sigma^{2}}\right) \exp \left(-\frac{v^{2}}{4 \sigma^{2}}\right) S(v) d v,
\end{aligned}
$$

where the 2 nd equality was found using the expression of $f$ of equation $(B .6$, and the 3rd equality by changing variables, writing $v_{\perp} d v_{\perp} d v_{\|}=v d v d v_{\|}$. 
For a linear decrease of $p_{\text {crit }}$ with $v$ (Roos \& Norman 1979), i.e. $S(v) \propto\left(v_{0}-v\right)^{2}$ (eq. [B.5]), the ratio of the rate of mergers with galaxies of cluster 2 to that with galaxies of the test galaxy's own cluster is found by integrating equation (B.7) and dividing by the integral (Mamon 1992) of equation (B.4):

$$
\begin{aligned}
\frac{k_{2}}{k_{1}} & =\frac{2 \sigma^{2}}{V} \exp \left(-\frac{V^{2}}{4 \sigma^{2}}\right) \frac{\int_{0}^{\infty} v^{2} \sinh \left(\frac{v V}{2 \sigma^{2}}\right) \exp \left(-\frac{v^{2}}{4 \sigma^{2}}\right) S(v) d v}{\int_{0}^{\infty} v^{3} \exp \left(-\frac{v^{2}}{4 \sigma^{2}}\right) S(v) d v} \\
& =\frac{\exp \left(-Y^{2}\right)}{2 Y} \frac{\int_{0}^{x} u^{2} \sinh (2 u Y) \exp \left(-u^{2}\right)(x-u)^{2} d u}{\int_{0}^{x} u^{3} \exp \left(-u^{2}\right)(x-u)^{2} d u} \\
& =\frac{\exp \left[-Y^{2}-x(x+2 Y)\right]}{32 Y\left[1-\exp \left(-x^{2}\right)+x^{2} / 2-(3 / 4) \sqrt{\pi} x \operatorname{erf}(x)\right]} F(x, Y),
\end{aligned}
$$

where $u=v /(2 \sigma), x=v_{0} /(2 \sigma), Y=V /(2 \sigma)$, and

$$
\begin{aligned}
F(x, Y)= & 2\left\{4 \exp [x(x+2 Y)] x^{2} Y+[\exp (4 x Y)-1] x\left(1+2 Y^{2}\right)\right. \\
& \left.-[1+\exp (4 x Y)-2 \exp [x(x+2 Y)]] Y\left(5+2 Y^{2}\right)\right\} \\
+ & \sqrt{\pi} \exp \left[(x+Y)^{2}\right] \\
\times & \left\{\left[3+4 Y^{2}\left(3+Y^{2}\right)-4 x Y\left(3+2 Y^{2}\right)+x^{2}\left(2+4 Y^{2}\right)\right] \operatorname{erf}(x-Y)\right. \\
& +2\left[3+2 x^{2}+4\left(3+x^{2}\right) Y^{2}+4 Y^{4}\right] \operatorname{erf}(Y) \\
& \left.\times\left[3+2 x^{2}+12 x Y+4\left(3+x^{2}\right) Y^{2}+8 x Y^{3}+4 Y^{4}\right] \operatorname{erf}(x+Y)\right\} .
\end{aligned}
$$

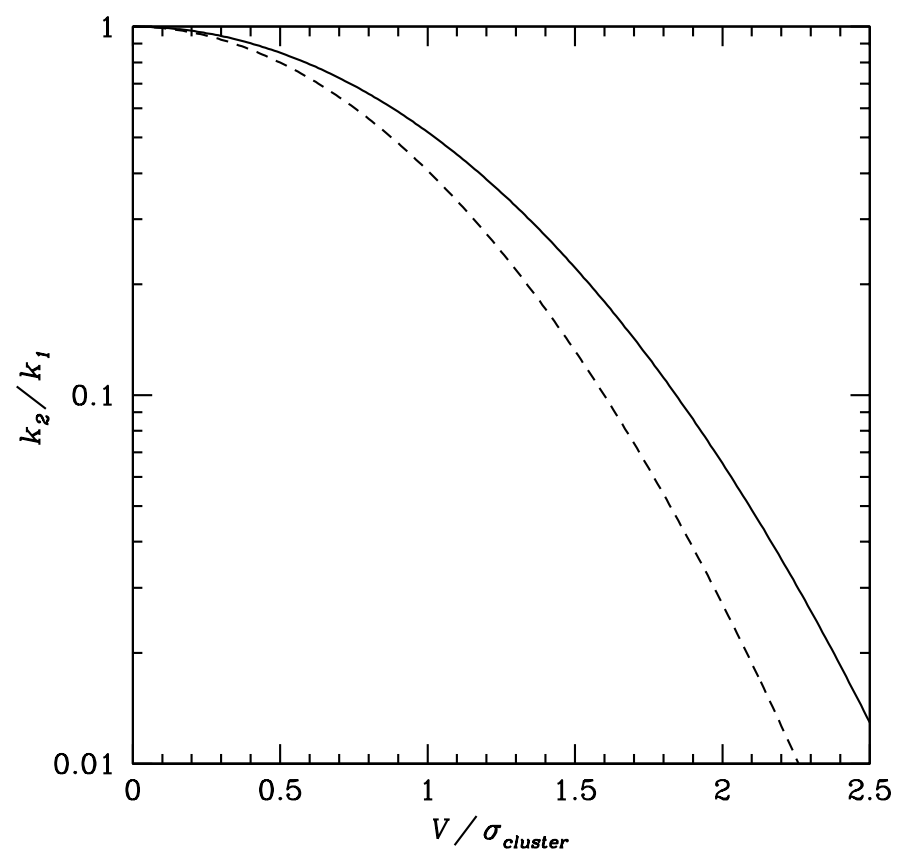

Fig. B.1. Ratio of the rates of direct mergers of a galaxy in a cluster with velocity dispersion $\sigma$ with galaxies of another equal mass cluster passing right through the first cluster at velocity $V$ to that of mergers with galaxies of its own cluster (eqs. [B.8] and [B.9]). The merger cross section (eq. [B.5]) involves a critical impact parameter that falls linearly with pericenter velocity, with a maximum velocity $v_{0}=3.1 \sqrt{3} \sigma_{g}$ (Roos \& Norman 1979), where $\sigma_{g}$ is the galaxy velocity dispersion, equal to $1 / 4$ (solid curve) or 1/8 (dashed curve) times the cluster velocity dispersion.

Figure B.1 displays $k_{2} / k_{1}$ as a function of $V / \sigma$ for two values of $\sigma_{g} / \sigma$ for the linear critical parameter versus velocity, with maximum merger velocity $v_{0}=3.1 \sqrt{3} \sigma_{g}$ (Roos \& Norman 1979). 


\section{References}

Abell G. O., Corwin H. G., Jr., Olowin R. P., 1989, ApJS, 70, 1

Bahcall N. A., Chokshi A., 1992, ApJ, 385, L33

Bardelli S., Zucca E., Baldi A., 2001, MNRAS, 320, 387

Bardelli S., Zucca E., Zamorani G., Moscardini L., Scaramella R., 2000, MNRAS, 312,540

Bardelli S., Zucca E., Zamorani G., Vettolani G., Scaramella R., 1998, MNRAS, 296, 599

Barnes J. E., 1988, ApJ, 331, 699

Becker R. H., White R. L., Helfand D. J., 1995, ApJ, 450, 559

Bekki K., Couch W. J., 2003, ApJ, 596, L13

Best P. N., 2004, MNRAS, 351, 70

Best P. N., von der Linden A., Kauffmann G., Heckman T. M., Kaiser C. R., 2007, MNRAS, 379, 894

Brand K., Rawlings S., Hill G. J., et al., 2003, MNRAS, 344, 283

Burns J. O., 1990, AJ, 99, 14

Carlberg R. G., Yee H. K. C., Ellingson E., 1997, ApJ, 478, 462

Colless M., Dalton G., Maddox S., et al., 2001, MNRAS, 328, 1039

Condon J. J., 1992, ARA\&A, 30, 575

Condon J. J., Cotton W. D., Greisen E. W., et al., 1998, AJ, 115, 1693

Coziol R., Ribeiro A. L. B., de Carvalho R. R., Capelato H. V., 1998, ApJ, 493, 563

Cutri R., Skrutskie M., Van Dyk S., et al., 2006, 2MASS Explanatory Supplement, Technical report, 2MASSExpSuppl

Di Matteo P., Combes F., Melchior A.-L., Semelin B., 2007, A\&A, 468, 61

Dressler A., Thompson I. B., Shectman S. A., 1985, ApJ, 288, 481

Drinkwater M. J., Parker Q. A., Proust D., Slezak E., Quintana H., 2004, PASA, 21,89

Fabian A. C., 1991, MNRAS, 253, 29P

Fabian A. C., Arnaud K. A., Nulsen P. E. J., Mushotzky R. F., 1986, ApJ, 305, 9

Gastaldello F., Ettori S., Molendi S., et al., 2003, A\&A, 411, 21

Gavazzi G., Jaffe W., 1986, ApJ, 310, 53

Gerin M., Combes F., Athanassoula E., 1990, A\&A, 230, 37

Gill S. P. D., Knebe A., Gibson B. K., 2005, MNRAS, 356, 1327

Gunn J. E., Gott J. R., 1972, ApJ, 176, 1

Hill G. J., Lilly S. J., 1991, ApJ, 367, 1

Jarrett T. H., Chester T., Cutri R., et al., 2000, AJ, 119, 2498

Jones D. H., Saunders W., Colless M., et al., 2004, MNRAS, 355, 747

Joseph R. D., Wright G. S., 1985, MNRAS, 214, 87

Kaldare R., Colless M., Raychaudhury S., Peterson B. A., 2003, MNRAS, 339, 652

Ledlow M. J., Owen F. N., 1996, AJ, 112, 9

Łokas E. L., Mamon G. A., 2001, MNRAS, 321, 155

Machalski J., Godlowski W., 2000, A\&A, 360, 463

Magliocchetti M., Maddox S. J., Hawkins E., et al., 2004, MNRAS, 350, 1485

Mamon G. A., 1992, ApJ, 401, L3

Mamon G. A., 2000, in 15th IAP Astrophys. Mtg., Dynamics of Galaxies: from the early Universe to the Present, ed. F. Combes, G. A. Mamon \& V. Charmandaris, Vol. 197. (ASP, San Francisco), p. 377, arXiv:astro-ph/9911333

Mamon G. A., Łokas E. L., 2005, MNRAS, 362, 95

Mamon G. A., Sanchis T., Salvador-Solé E., Solanes J. M., 2004, A\&A, 414, 445

Miller N. A., 2005, AJ, 130, 2541

Miller N. A., Owen F. N., 2001, ApJS, 134, 355

Miller N. A., Owen F. N., 2002, AJ, 124, 2453

Miller N. A., Owen F. N., 2003, AJ, 125, 2427

Monaco P., Giuricin G., Mardirossian F., Mezzetti M., 1994, ApJ, 436, 576

Navarro J. F., Frenk C. S., White S. D. M., 1996, ApJ, 462, 563

Owen F. N., Ledlow M. J., Keel W. C., Morrison G. E., 1999, AJ, 118, 633

Peacock J. A., Nicholson D., 1991, MNRAS, 253, 307

Pietsch W., Trinchieri G., Arp H., Sulentic J. W., 1997, A\&A, 322, 89

Poggianti B. M., 1997, A\&AS, 122, 399

Prestage R. M., Peacock J. A., 1988, MNRAS, 230, 131

Proust D., Quintana H., Carrasco E. R., et al., 2006, A\&A, 447, 133

Quintana H., Carrasco E. R., Reisenegger A., 2000, AJ, 120, 511

Quintana H., Ramirez A., Melnick J., Raychaudhury S., Slezak E., 1995, AJ, 110,463

Raychaudhury S., 1990, Ph.D. thesis, Univ. Cambridge

Raychaudhury S., Fabian A. C., Edge A. C., Jones C., Forman W., 1991, MNRAS, 248, 101

Reisenegger A., Quintana H., Carrasco E. R., Maze J., 2000, AJ, 120, 523

Roettiger K., Burns J., Loken C., 1993, ApJ, 407, L53

Roos N., 1981, A\&A, 104, 218

Roos N., Norman C. A., 1979, A\&A, 76, 75

Sadler E. M., Jackson C. A., Cannon R. D., et al., 2002, MNRAS, 329, 227

Sanderson A. J. R., Ponman T. J., O’Sullivan E., 2006, MNRAS, 372, 1496
Strauss M. A., Weinberg D. H., Lupton R. H., et al., 2002, AJ, 124, 1810

Tully R. B., 1988, AJ, 96, 73

van der Hulst J. M., Rots A. H., 1981, AJ, 86, 1775

Venturi T., Bardelli S., Morganti R., Hunstead R. W., 1997, MNRAS, 285, 898

Venturi T., Bardelli S., Morganti R., Hunstead R. W., 2000, MNRAS, 314, 594

Venturi T., Bardelli S., Zambelli G., Morganti R., Hunstead R. W., 2001, MNRAS, 324, 1131

Yun M. S., Reddy N. A., Condon J. J., 2001, ApJ, 554, 803

\section{List of Objects}

'Abell 3558' on page 2

'Abell 3528' on page 2 